\title{
Evaluación de productos IMERG V03 y TMPA V7 en la detección de crecidas caso de estudio cuenca del río Cañar
}

\author{
Guachamín Wilmer',2, Páez-Bimos Sebastián², Horna Natalia² \\ ${ }^{1}$ Instituto Nacional de Meteorología e Hidrología \\ ${ }^{2}$ Escuela Politécnica Nacional, Facultad de Ingeniería Civil y Ambiental, Quito, Ecuador
}

\begin{abstract}
Resumen: Los productos de precipitación por satélite de la Misión de Medición de Precipitación Tropical (TRMM) y su sucesor la Medición de Precipitación Global (GPM), proveen de datos de precipitación para aplicaciones hidrológicas en cuencas hidrográficas sin datos o información escasa. El propósito de este estudio es evaluar la aplicación de los productos satelitales IMERG V03 y TMPA V7 para modelación hidrológica y la potencial detección de caudales de crecidas en la cuenca del río Cañar. Los productos satelitales IMERG V03 y TMPA V7 a escala espacio temporal $0.1^{\circ} \times 0.1^{\circ}(10 \times 10 \mathrm{~km}) / 1$ hora y $0.25^{\circ} \times 0.25^{\circ}(25 \times 25 \mathrm{~km}) / 3$ horas respectivamente, en eventos de crecidas en el período marzo 2014 a diciembre 2015 subestiman las intensidades de precipitación, misma que se atribuye a la topografía fuertemente accidentada. Los resultados muestran que los productos satelitales mejoran la distribución espacial de la lluvia registrada solamente con los pluviómetros considerando los métodos de corrección como Double Kernel Smoothing (DS), y Residual Inverse Distance Weigthing (RIDW). Para modelación hidrológica con HEC-HMS se pudo identificar que los productos de precipitación corregidos por los métodos DS y RIDW generan caudales más ajustados a los observados, especialmente cuando los eventos de crecida registran una alta probabilidad de detección de lluvia (POD) y una mayor intensidad de precipitación. Los resultados muestran el potencial que tienen productos satelitales fusionados con observaciones de campo para la simulación de caudales de crecidas en cuencas con escasos datos de campo.
\end{abstract}

Palabras clave: IMERG, TMPA, DS, RIDW, modelo, eventos de crecidas.

\section{Evaluation of IMERG V03 and TMPA V7 products in the detection of flood events in Cañar river basin}

\begin{abstract}
The satellite precipitation products, such as Tropical Rainfall Measuring (TRMM) and it is successor Global Precipitation Measurement (GPM), provide precipitation data for hydrological applications in basins without data or with scarce information. The purpose of this paper is to evaluate satellite-based products IMERG V03 and TMPA V7, for hydrological modeling and the potential detection of flood discharges in the Cañar river basin. The evaluation of IMERG V03 and TMPA V7 satellite products at a different spatio-temporal resolutions of $0.1^{\circ} \mathrm{x} 0.1^{\circ}$ $(10 \times 10 \mathrm{~km}) 1$ hour, and $0.25^{\circ} \times 0.25^{\circ}(25 \times 25 \mathrm{~km}) 3$ hours respectively, at events during the period March $2014-$ December 2015, shows that the rainfall intensity is underestimated. This is related to the disperse rain gages, which do not register in a proper manner the rainfall events, as well as the mountainous topography of the terrain. The results show that the satellite-based products improve the rainfall spatial distribution registered by rain gages, when including correction methods such as Double Kernel Smoothing (DS) and Residual Inverse Distance Weighting (RIDW). The evaluation of the correction methods was carried out by comparing discharges simulated with the hydrological model HEC-HMS and observed ones. When applying the DS and RIDW methods to IMERG V03 and TMPA V7 products, the hydrological model is able to produce discharges that are closer to the observed ones. Moreover, the calibrated hydrologic model HEC-HMS can simulate discharges that fit the flood events for all both satellite-based products (IMERG V03 and TMPA V7), when these products register a high probability for detection (POD) and a high rainfall intensity. The results show the potential of satellite products merged with rainfall observations for the simulation of flood discharges in basins with scarce rainfall data.
\end{abstract}

Keywords: IMERG, TMPA, DS, RIDW, modelling, flood events.

\section{INTRODUCCIÓN}

La información de precipitación es fundamental como entrada para la simulación de caudales a través de modelos hidrológicos. Las simulación hidrológica es una tarea desafiante especialmente en regiones montañosas donde la red pluviométrica es escasa y donde existe una gran variabilidad espacial de precipitación producto de relieve de alta montaña (Buytaert et al., 2006; Castro, 2016). 
En la actualidad se encuentran disponibles productos satelitales derivados de la misión de Medición de Precipitación Tropical (TRMM) y su sucesora, la misión de Medición de Precipitación Global (GPM), los cuales proveen datos a escala global y generalmente a altas resoluciones espacio-temporales. Estas proporcionan una alternativa de fuente de datos para la variable precipitación en cuencas hidrográficas con escasez de datos o sin datos.

Uno de los últimos avances en el campo de la teledetección para estimar la precipitación a escala global es la misión GPM, lanzada por la National Aeronautics and Space Administration (NASA) y Japan Aerospace Exploration Agency (JAXA) el 27 de febrero de 2014, diseñada para abordar las limitaciones de su predecesora la misión TRMM. La misión GPM establece nuevos estándares para las mediciones de precipitación, mejorando las resoluciones espacio temporales y estimaciones de nevadas así como lluvias ligeras gracias a la mejora en sus sensores pasivos y activos (Liu et al., 2015).

La estimación y predicción de fuertes precipitaciones es crucial para muchas aplicaciones, tales como la gestión de recursos hídricos, predicción de inundaciones, alerta temprana; así como para la gestión y mitigación de desastres (Prakash et al., 2016). Las inundaciones repentinas mantienen un rango devastador y son responsables de daños significativos, pérdidas de vidas e impacto económico. Esta amenaza requiere el desarrollo de sistemas eficaces de alerta de inundaciones para mitigar el riesgo. Los sistemas de alerta de inundaciones en base a mediciones de lluvia o estimaciones de teledetección con modelos hidrológicos pueden proporcionar predicciones de caudales de crecidas (Nikolopoulus et al., 2013).

En referencia a investigaciones sobre inundaciones en la región, Sedano et al., (2013) en el Análisis de aspectos que incrementan el riesgo de inundaciones en Colombia, para el periodo abril 2010 a diciembre 2011 en el que se registró 5.2 millones de personas afectadas y 683 muertos, concluyen que es necesario abordar el manejo de inundaciones con un enfoque diferente, migrando hacia procesos integrales y con mayor capacidad de adaptación. Ávila et al., (2016) en el estudio de tendencias recientes de las precipitaciones, inundaciones repentinas y deslizamientos de tierra en el sur de Brasil indican que la intensidad de las precipitaciones ha aumentado en las escalas de tiempo anuales y estacionales, aunque estas no alcanzan umbrales estadísticos significativos sus impactos en el medio ambiente son severos con un incremento en deslizamientos de tierra y produciendo inundaciones repentinas. Además indica que estos resultados pueden ser utilizados por los tomadores de decisiones para establecer umbrales iniciales en medidas preventivas. Jha et al., (2012) ofrecen una orientación operativa de gestión de riesgo de inundaciones en un entorno de transformación del medio ambiente y clima cambiante en el que se identifican medidas adecuadas e integradas en materia de riesgo de inundaciones. Adicionalmente en uno de sus casos de estudios indica sobre la Misión de Medición de Precipitaciones (TRMM), menciona que ha mejorado la modelización de precipitaciones mismo que han conducido a un mejor pronóstico de las inundaciones.
En cuanto a lo modelación hidrológica de crecidas en cuencas se puede mencionar a estudios como, Arteaga (2017) donde se evalúa el modelo hidrológico HEC-HMS para la predicción hidrológica y de crecidas, en la cuenca baja del río Cañar, validando el modelo calibrado con cuatro eventos registrados, dos eventos de crecida fueron reproducidos satisfactoriamente por lo que recomienda analizar a mayor detalle los datos hidrometeorológicos y características físicas de la cuenca antes de su aplicación. Otros estudios a nivel global como Joo et al., (2013) presenta un estudio comparativo de dos modelos lluvia escorrentía ReFH-model y HEC-HMS en eventos de inundación en Corea, el modelo ReFH mostró limitaciones en la simulación de caudal máximo mientras que HEC-HMS mostró mejor caudal máximo. Razi et al., (2010) realizarron un estudio de estimación de inundaciones utilizando el modelo HEC-HMS para el río Johor en Malasia, la evaluación del rendimiento del modelo registra un coeficiente de correlación cercano a 1 , el error del $4 \%$ del caudal pico con lo que sugiere que este modelo se puede utilizar como herramienta para estimación de caudal pico.

En la modelación hidrológica HEC-HMS a partir de productos satelitales se la ha realizado en diversas cuencas con fines de estimación de crecidas. Nikolopoulos et al., (2013) destacan la necesidad de mejorar los productos de precipitación satelital para permitir un uso más apropiado para aplicaciones de eventos de inundación. Shahid et al., (2016) evalúa el modelo HEC-HMS para simular caudales basado en datos de precipitación de la misión TRMM, el modelo calibrado fue evaluado y se encontró consistencia en caudales simulados y observados. Por tanto la información satelital pudo ser útil para proporcionar alertas de picos de caudal en tiempo real. Yang et al., (2017), sobre la aplicabilidad de la precipitación obtenida en base a productos de la misión TRMM por el modelo hidrológico HEC-HMS para la identificación de inundaciones en la cuenca del río Xiangjiang en China, indica que los resultados de simulación son aceptables para identificar eventos a pesar del sesgo en el tiempo y magnitud de caudales picos, por lo que indica que las simulaciones deben ser usados con precaución. Los dos últimos casos muestran resultados alentadores en el contexto de cuencas de gran tamaño.

En cuanto a la evaluación de productos satelitales y la modelación hidrológica en contexto regional, Zulkafli et al., (2013), en el análisis comparativo de rendimiento de las versiones 6 y 7 de TRMM 3B42 (TMPA) para aplicaciones hidrológicas en cuencas andino-amazónicas, concluyen que el producto de la versión 7 tiene un sesgo significativamente más bajo y una mejor representación de la distribución de las precipitaciones. Además de las versiones 6 y 7 para modelación hidrológica la mejora en el algoritmo de estimación de precipitación de la versión 7 se traduce en aumento de eficiencia del Nash-Sutclife y reducción del sesgo relativo entre caudales observados y simulados en un 30\%95\%. Méndez et al., (2016), para la estimación de precipitación obtenida de productos satelitales de alta resolución espaciotemporal, con registros pluviométricos a escala diaria y técnicas de corrección, muestran que IMERG presenta mejor correlación que CMORPH y CHIRPS sin embargo IMERG presenta mayor subestimaciones de 
precipitación para altitudes medias seguido por CMORPH y CHIRPS. Zubieta et al., (2017), en el estudio de productos de precipitación IMERG versión final, como datos de entrada a un modelo hidrológico distribuido para la cuenca amazónica de Perú y Ecuador, así como los productos TRMM (TMPA V7, TMPA RT) y datos de lluvia observada. En general, los autores concluyen que IMERG, TMPA V7 y TMPA RT se correlaciona con la precipitación observada y para el análisis de caudales indica que IMERG es tan útil como TMPA V7 y TMPA RT. Recientemente, Asurza et al., (2018) para la evaluación de productos 3B42RT, 3B42V7 e IMERG en el modelamiento hidrológico de una cuenca en el altiplano Peruano indica que IMERG corregido presenta una considerable mejora permitiendo captar muy bien la estacionalidad de la lluvia reduciendo la subestimación. En consecuencia su aplicación en el modelamiento hidrológico permite estimar mejor los caudales.

En el Ecuador el INAMHI ha implementado un sistema de alerta temprana para la cuenca del río Cañar (SAT Cañar) con 13 estaciones hidro-meteorológicas que registran variables en tiempo real y transmiten a intervalo de tiempo horario, los caudales y precipitación desde el fin del año 2013. Sin embargo, a pesar de los esfuerzos realizados aún quedan cuencas que no cuenta con instrumentación adecuada; por lo que, el estudio sobre la aplicación de sensores remotos en la detección de crecidas es una alternativa válida para complementar la limitación de datos provenientes de pluviómetros.

En la región hasta el momento no se han publicado resultados de modelación de caudales de crecida en base a productos de precipitación derivados de sensores de teledetección; sin embargo, su estudio y análisis ha sido sugerido (Zubieta et al., 2017). Planteado ese contexto general, esta investigación tiene por objetivo evaluar los productos satelitales IMERG V03 y TMPA V7 como entrada de precipitación para modelación hidrológica de caudales de crecidas en la cuenca del río Cañar.

\section{METODOLOGÍA}

\subsection{Zona de estudio}

La cuenca hidrográfica del Cañar $\left(2246 \mathrm{~km}^{2}\right)$ se ubica en la zona centro occidental del Ecuador. La cuenca alta tiene una topografía fuertemente accidentada conformada, por la cordillera occidental de los Andes, y descendiendo abruptamente la elevación del terreno hasta llegar a una topografía plana en el punto de cierre de la cuenca a $19 \mathrm{msnm}$. (Figura 1).

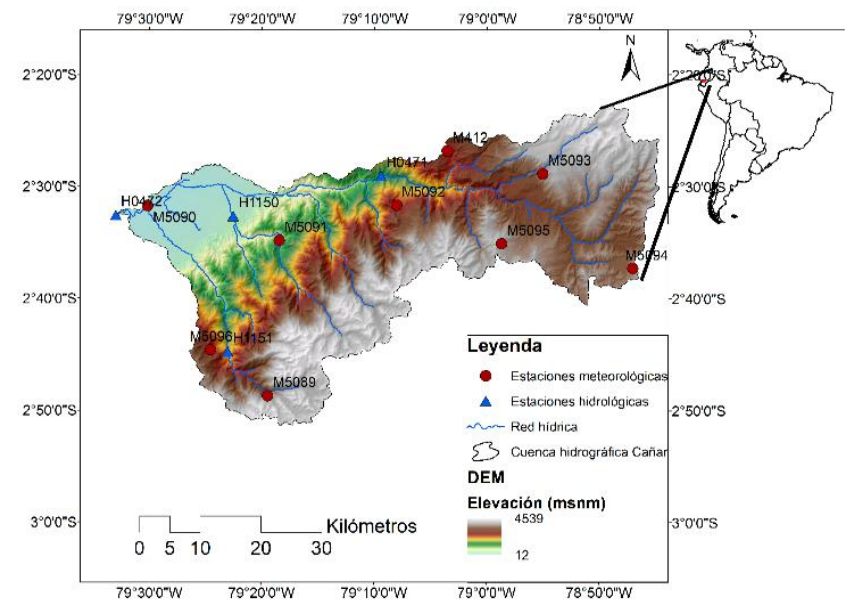

Figura 1. Ubicación del área de estudio

En la cuenca se producen frecuentemente crecidas que afectan a la población aledaña. Esto ha llevado que las autoridades locales y regionales pongan en marcha diferentes actividades para mitigar su amenaza. Es así que Secretaria Nacional del Agua en el año 2009 contrato los estudios para el control de inundaciones del río Bulubulu, Cañar y Naranjal cuyo objetivo fue medidas ingenieriles para controlar las inundaciones que producen estos ríos y estabilizar sus cauces. La obra se inauguró en el 2016, a partir de este año mitigar las crecidas del río Cañar debido a intensidades fuertes. De esta forma se busca proteger poblados, infraestructura y áreas de producción agrícola. Es importante que estas medidas estructurales vayan acompañadas de medidas no estructurales como sistemas de alerta temprana en fin de complementar la protección ante inundaciones.

En la Figura 2, se muestra el marco metodológico que describe el proceso mediante el cual se procura obtener información para entender, verificar, corregir o aplicar los productos de precipitación IMERG V03 y TMPA V7 en la detección de caudales de crecida en la cuenca del río Cañar. 


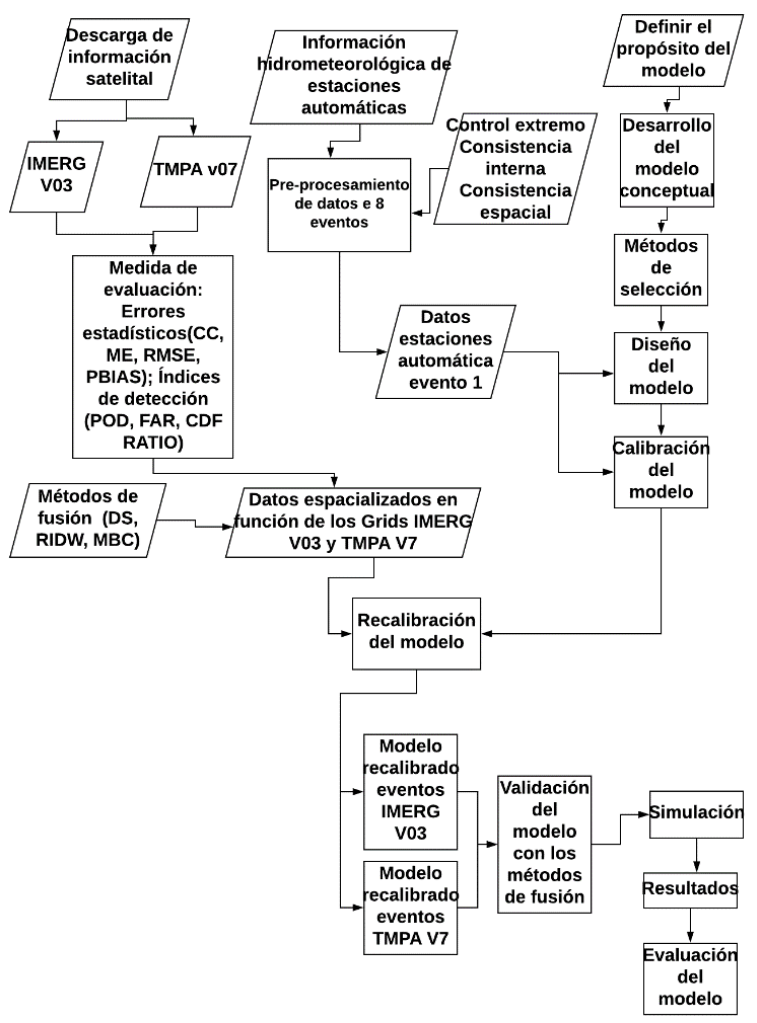

Figura 2. Flujo de procesos en la determinación de caudal

\subsection{Recopilación y procesamiento de información}

Para este estudio se ha recopilado información, requerida y disponible de tipo hidrometeorológica, series a menor intervalo de tiempo disponible de estaciones automáticas y convencionales del INAMHI, cartográfica de la red hidrometeorológica, textura de suelos, cobertura vegetal de instituciones gubernamentales (Tabla 1). En lo correspondiente a información satelital, se utilizaron los datos de los productos IMERG V03 y TMPA V7 a escala espacio temporal de $0.1^{\circ} \mathrm{x} 0.1^{\circ}$ a 30 minutos y $0.25^{\circ}$ x $0.25^{\circ}$ a 3 horas, respectivamente. Ambos productos son generados por la NASA y obtenidos de https://pmm.nasa.gov/.

\subsection{Preprocesamiento de datos precipitación}

El preprocesamiento de la información de lluvia se ha realizado por medio de tres métodos: control de extremos, consistencia interna y consistencia espacial (Shen et al. 2010; Manz et al. 2017). Para el control extremo se comparan valores horarios máximos con los diarios, de las estaciones automáticas con valores diarios máximos obtenidos de estaciones convencionales. Si el dato horario supera el valor diario esta debe ser reemplazada como no disponible (NA). Del análisis de estaciones automáticas en la cuenca hidrográfica del río Cañar, en comparación con estaciones convencionales se lo realizó únicamente para la estación M0412 Suscalpamba de este control de datos se obtuvo un $0.37 \%$ de datos rechazados o no válidos.

Para el resto de estaciones se realizó un análisis de consistencia interna (Figura 3), donde se eliminaron valores de las estaciones automáticas H0471, M5091, M115 que excedían valores de precipitación máxima en 24 horas del estudio de lluvias intensas (Guachamín, 2015), identificados en las Zonas 15,20 y 47 respectivamente, registrando el porcentaje más alto para la estación H0471 con $6.58 \%$.

Tabla 1. Información utilizada en el estudio.

\begin{tabular}{|c|c|c|c|c|c|}
\hline Información cartográfica & Formato & Año & Resolución/ Escala & Descripción & Fuente \\
\hline SRTM & Raster & 2009 & $30 \times 30 \mathrm{~m}$ & Modelo digital de terreno $30 \mathrm{~m}$. & NASA \\
\hline Estaciones meteorológicas & Vector & 2014 & $1: 50000$ & Ubicación de estaciones hidrometeorológicas & INAMHI \\
\hline Textura del suelo & Vector & 2002 & $1: 250000$ & Descripción de texturas del suelo & MAGAP-SIGAGRO \\
\hline Cobertura y uso del suelo & Vector & 2013-2014 & $1: 100000$ & Caracterización de uso y cobertura del suelo & MAE-MAGAP \\
\hline
\end{tabular}

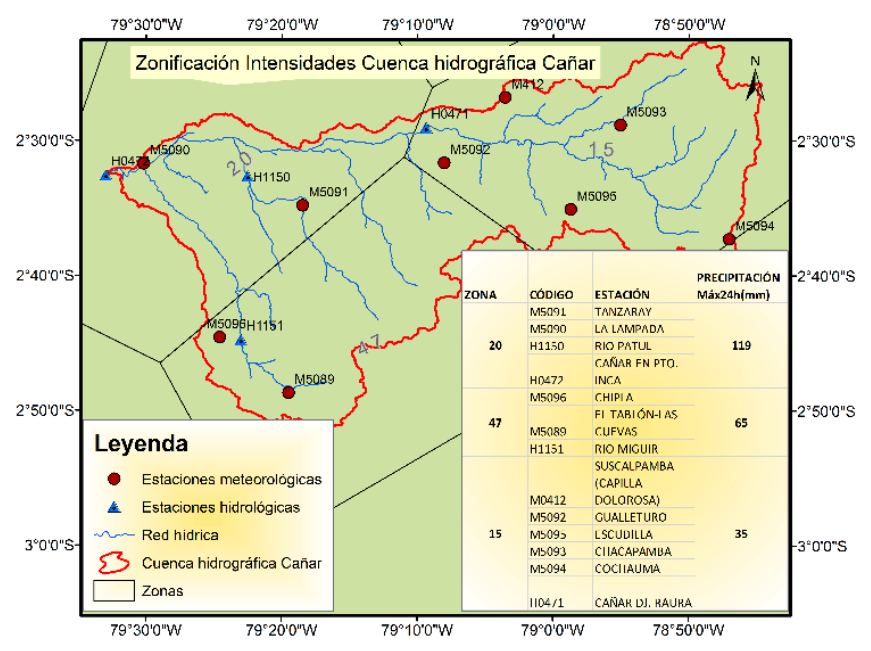

Figura 3. Zonificación de intensidades
En cuanto a consistencia espacial se realizó una revisión manual a través de los gráficos de la serie temporal para cada estación automática de la cuenca hidrográfica en estudio. Para lo cual se consideró la situación altitudinal de las estaciones automáticas y la cercanía entre las mismas. Se registraron valores altos para las estaciones M1151, M5096 y M0412 valores que fueron rechazados y reemplazados por NA en la base de datos.

\subsection{Preprocesamiento de datos caudal}

La estación hidrológica H0472 Puerto Inca localizada a la salida de la cuenca hidrográfica, es la estación que registró los datos más confiables de nivel de agua en el río Cañar. Para estos datos se revisó el ajuste gráfico de los hidrogramas en sus valores máximo, medio y mínimo instantáneos en base a observaciones diarias, por lo que se convierte en la estación de control para este estudio. 


\subsection{Descripción de productos satelitales de precipitación}

\section{TMPA}

El producto TRMM Análisis de Precipitación Multisatélite (TMPA), se basa en la calibración de estimaciones de precipitación, obtenidas mediante sensores microonda e infrarojos de múltiple satélites, que luego son ajustadas con observaciones de precipitación. El resultado de este proceso es el producto tipo de investigación (TMPA "research" versión), que se produce a una resolución espacio-temporal de $0.25^{\circ} \mathrm{x}$ $0.25^{\circ}$ y 3 horas. El conjunto de datos cubre una banda de lat/lon $50^{\circ} \mathrm{N}-\mathrm{S}$ para el período desde 1998 a la actualidad. (Huffman et al. 2007).

\section{IMERG}

El producto IMERG se basa en una red internacional de satélites, que proporcionan estimaciones de precipitación de alta resolución, combinando mediciones de varios sensores microondas (PWM) e infrarrojos (IR) (Huffman et al. 2015). Los algoritmos para la producción de IMERG provienen de algoritmos que fueron desarrollados para la misión TRMM, así como de otros desarrollados para productos similares.

Este estudio se centra en el algoritmo de IMERG V03 que intercalibra todas las estimaciones de precipitación de microondas, junto con estimaciones de sensores infrarrojos calibrados por microondas (Huffman et al. 2015) en una resolución espacial de $0.1^{\circ}$ x $0.1^{\circ}$ y una resolución temporal de 30 minutos. El conjunto de datos cubre una banda de lat/lon $60^{\circ} \mathrm{N}$-S para el periodo marzo 2014 a la actualidad. El sistema se ejecuta dando primero una estimación rápida y proporcionando sucesivamente mejores estimaciones a medida que llegan más datos. El paso final usa datos de pluviómetros mensuales para crear productos a nivel de investigación (Huffman et al. 2015). Los sensores del GPM son el microwave imager (GMI) y radar de precipitación de doble frecuencia (DPR). El sensor GMI presenta resolución espacial más alta que el TRMM microondas imager (TMI), mientras que el sensor DPR presenta mejor exactitud en mediciones y mejor identificación de líquidos, hielo, partícula de precipitación de fase mixta en comparación con el sensor radar de precipitación (PR) del TRMM.

La base de datos de lluvia a nivel horario de estaciones automáticas del INAMHI en la cuenca del río Cañar fue la información de referencia para el ejercicio comparativo, ajuste y corrección, tomando como referencias algunas investigaciones planteadas (Nerini et al. 2015; Manz et al. 2017). La resolución espacio-temporal de productos para este estudio correspondiente IMERGV03 es $0.1^{\circ} 1$ hora y a TMPA es $0.25^{\circ} 3$ horas.

\subsection{Selección y análisis de eventos}

La información de la estación automática Cañar en Puerto Inca (H0472) está disponibles desde el año 2013 a la presente fecha. Sin embargo, la información de productos IMERG está disponibles desde febrero de 2014 hasta la fecha. Adicionalmente, el 12 de enero de 2016 se inauguró el sistema de control de inundaciones Cañar; por lo que la información registrada por la estación $\mathrm{H} 0472$ a partir de esta fecha refleja caudales no naturales. Por esta razón se consideró el rango de información de 12/03/2014 al 31/12/2015.

Se han definido umbrales de inundación en las estaciones automáticas meteorológicas e hidrológicas en base a los registros históricos de las estaciones convencionales y en los análisis de modelación hidrológica e hidrodinámica. Este análisis se validó también a la experiencia de los observadores de las estaciones hidrológicas automáticas y convencionales las cuales fueron comprobadas con los registros históricos de nivel. Se consideró un evento cuando el nivel de agua en la estación hidrológica Cañar en Pto. Inca (H0472) supera su umbral de aviso de $3.5 \mathrm{~m}$. La estación Cañar en Pto. Inca (H0472) ha sido considerada como la estación de control, debido a que se encuentra ubicada aguas abajo en el area propensa a inundaciones, misma que registra información automatica horaria de nivel de agua. El umbral de alerta $4.5 \mathrm{~m}$ está definido en función del inicio de la inundación, mientras que el umbral de aviso es cercano al $75 \%$ del valor de alerta (Páez \& Llerena, 2014).

En este estudio se registran ocho eventos que superan el nivel de aviso y tan solo uno (evento 1) supera el nivel de alerta. Para la modelación se consideró el evento 1 al ser el más representativo de una crecida. Los otros eventos solo superaron el nivel del umbral de aviso, por lo que no fueron representativos para calibrar o validar un evento de crecida. Eso se confirma al comparar los caudales máximos de los eventos 2 al 8 , que son alrededor de la mitad del caudal máximo del evento 1 . Por esta razón, se ha seleccionado para la validación de caudales solo el evento 5, ya que es el que mejores resultados de modelación presentaba y el cual se encontraba dentro de los eventos más cercanos al nivel de alerta. En cuanto a la evaluación de la precipitación, si se consideraron los 8 eventos registrados en la Tabla 2.

Tabla2. Eventos ocurridos en la estación H0472.

\begin{tabular}{|c|c|c|c|c|c|}
\hline $\mathbf{N}^{0}$ & $\begin{array}{l}\text { Inicio } \\
\text { evento }\end{array}$ & Fin evento & $\begin{array}{l}\text { Tiempo al } \\
\text { pico }\end{array}$ & $\begin{array}{l}\text { Nivel } \\
\text { (m) }\end{array}$ & $\begin{array}{l}\text { Caudal } \\
\left(\mathrm{m}^{3} / \mathrm{s}\right)\end{array}$ \\
\hline \multirow[t]{2}{*}{1} & $29 / 03 / 2014$ & $31 / 03 / 2014$ & $30 / 03 / 2014$ & 5.55 & 725 \\
\hline & $12: 00$ & $12: 00$ & $15: 00$ & & \\
\hline \multirow[t]{2}{*}{2} & $06 / 02 / 2015$ & $10 / 02 / 2015$ & $09 / 02 / 2015$ & 3.55 & 277.8 \\
\hline & $22: 00$ & 02:00 & 07:00 & & \\
\hline \multirow[t]{2}{*}{3} & $24 / 02 / 2015$ & $25 / 02 / 2015$ & $24 / 02 / 2015$ & 4.48 & 436.4 \\
\hline & 00:00 & 05:00 & $10: 00$ & & \\
\hline \multirow[t]{2}{*}{4} & $19 / 03 / 2015$ & $21 / 03 / 2015$ & $20 / 03 / 2015$ & 4.19 & 383.9 \\
\hline & $16: 00$ & $12: 00$ & 9:00 & & \\
\hline \multirow[t]{2}{*}{5} & $27 / 03 / 2015$ & $30 / 03 / 2015$ & $29 / 03 / 2015$ & 4.08 & 364.8 \\
\hline & 16.00 & $18: 00$ & $5: 00$ & & \\
\hline \multirow[t]{2}{*}{6} & $16 / 04 / 2015$ & $17 / 04 / 2015$ & $17 / 04 / 2015$ & 3.94 & 341.1 \\
\hline & $0: 00$ & $19: 00$ & $0: 00$ & & \\
\hline \multirow[t]{2}{*}{7} & $25 / 04 / 2015$ & $27 / 04 / 2015$ & $26 / 04 / 2015$ & 3.70 & 302.2 \\
\hline & 1:00 & 08:00 & $22: 00$ & & \\
\hline \multirow[t]{2}{*}{8} & $05 / 05 / 2015$ & $06 / 05 / 2015$ & $06 / 05 / 2015$ & 3.46 & 265.5 \\
\hline & $2: 00$ & $15: 00$ & $1: 00$ & & \\
\hline
\end{tabular}

\subsection{Medidas de evaluación de precipitación}

La evaluación de los productos satelitales en base a las observaciones de campo a nivel 1 hora y 3 horas, se ha realizado en dos categorías. La primera, cuantitativa, describe la relación de estimaciones satelitales y las observaciones 
mediante indicadores estadísticos como coeficiente de correlación (CC), Error medio absoluto (MAE), Raíz del error medio cuadrático (RSME), Sesgo relativo (PBIAS). La segunda categoría, cualitativa, se utiliza para describir la habilidad de los productos satelitales en la detección de la lluvia mediante indicadores como la probabilidad de detección (POD), Falsa alarma (FAR), CDF Ratio (Tang et al. 2016; Tan et al. 2016; Prakash et al. 2016).

El insumo para el cálculo de los indicadores estadísticos es la información de IMERG V03 $0.1^{\circ} \mathrm{x} 0.1^{\circ}$ a 1 hora y TMPA V7 $0.25^{\circ} \times 0.25^{\circ}$ a 3 horas, con valores observados de las estaciones automáticas en el área de estudio.

La base de datos de precipitaciones de estaciones del INAMHI organizada fue la información de referencia para el ejercicio comparativo, ajuste y corrección, a la resolución temporal tomando como referencias algunas investigaciones planteadas (Nerini et al. 2015).

\subsection{Errores estadísticos}

Las medidas estadísticas que fueron utilizadas en este estudio son:

Coeficiente de Correlación (CC), calcula el nivel de asociación entre datos de información satelital y los pluviómetros, el valor del coeficiente está $-1 \leq 1$. Donde $\mathrm{CC}=1$ indica correlación positiva perfecta.

$$
C C=\frac{\sum_{i=1}^{n}(S i-\bar{S})(G i-\bar{G})}{\sqrt{\sum_{i=1}^{n}(S i-\bar{S})^{2}} \sqrt{\sum_{i=1}^{n}(G i-\bar{G})^{2}}}
$$

Error medio absoluto (MAE), toma el valor absoluto de los errores y los promedia durante todas las series de tiempo consideradas, toma el valor absoluto de cada número para de esta manera evitar que el número negativo y números positivos se anulen, valor perfecto del MAE es 0 .

$$
M A E=\frac{1}{N} \sum_{n=1}^{N}(S i-G i)
$$

La raíz del error medio cuadrático (RMSE), evalúa la magnitud típica del error de las estimaciones del satélite con la de los pluviómetros con sensibilidad a los errores más grandes y valores atípicos, valor perfecto del RMSE es 0 .

$$
R M S E=\sqrt{\frac{1}{n} \sum_{1}^{n}(S i-G)^{2}}
$$

El sesgo relativo (PBIAS), evalúa la diferencia media entre las estimaciones del producto satelital y la observada pluviométrica. El sesgo relativo puede ser positivo o negativo. Un sesgo negativo indica una subestimación de la precipitación satelital y un sesgo positivo una sobreestimación de precipitación satelital, el valor perfecto es 0 .

$$
\text { PBIAS }=\frac{\sum_{i=1}^{n}(S i-G i)}{\sum_{i=1}^{n} G i} x 100 \%
$$

Donde $S i, G i$ son intensidad de precipitación del satélite y observada (pluviómetro) respectivamente en el paso de tiempo $i$ y $\bar{S} y \bar{G}$ son los correspondientes promedios aritméticos durante el período de evaluación y $n$ es el número total de pasos de tiempo.

\section{9 Índices de detección}

Para la detección de eventos de precipitación se calculó la: probabilidad de detección (POD), falsa alarma (FAR) y CDF RATIO. La evaluación de la estimación de satélite sobre pluviómetro se realiza mediante el uso de la tabla de contingencia que refleja la frecuencia de "si" o "no" el producto satelital y las estaciones registran precipitación.

Donde un acierto (a) se registra cuando el producto satelital y el pluvímetro registran datos. Una falsa alarma (b) se obtiene cuando el producto satelital registra un valor pero no en los valores de pluviómetro. Los valores (c) cuando no registra el producto satelital y si registra el pluviómetro y correctos negativos (d) se dan cuando no existe valor del producto satelital ni del pluviómetro.

Probabilidad de detección (POD), se define como la proporción de ocurrencias que se pronosticaron correctamente, mientras más cercano a 1 es el valor de POD mejor es la detección realizada por el satélite, una estimación perfecta POD es igual a 1 .

$$
P O D=\frac{a}{a+c}
$$

Falsa alarma (FAR), es la detección de precipitación que en realidad no sucedieron, es decir que detecto el satélite pero no los pluviómetros. El valor de este término está entre 0 y 1 mientras más cercano a 0 sea el valor quiere decir que mejor es el pronóstico realizado y que no se han emitido falsas alarmas.

$$
F A R=\frac{b}{a+b}
$$

CDF RATIO, determina la función empírica de distribución acumulativa $(C D F ; F(x))$ para cada producto de precipitación satelital (SPP), se compara con el correspondiente indicador de CDF. La CDF puede representarse como percentiles discretos, y la proporción de los percentiles a través de toda la CDF muestra lo bien que los SPP estiman el indicador de distribución de las lluvias. Una estimación perfecta de CDF RATIO es 1.

$$
\text { CDF RATIO }=\frac{P s, j}{P G, j}
$$

Dónde: $P$ precipitación correspondiente al percentil $j, S$ y $G$ son respectivamente intensidad de precipitación satelital y pluviómetro, una estimación perfecta de CDF RATIO es 1. 


\subsection{Métodos de fusión}

La fusión de valores de precipitación IMERG V03 1 hora y TMPA V7 3 horas, se realizó utilizando técnicas indicadas por Nerini et al., (2015) y Dinku et al., (2013). Estos métodos propuestos utilizan los datos de precipitación observada a nivel 1 hora y 3 horas. Para realizar esta fusión se utilizó la librería Rainmerging del Git Hub que se puede descargar de la dirección https://github.com/zedzulkafli/Rainmerging/tree/master/R.

Los métodos de fusión que se utilizaron de la librería fueron Mean Bias Correction (MBC), Double Kernel Smoothing (DS), Residual RIDW interpolation (RIDW).

Mean Bias Correction (MBC), método de fusión basado en productos satelitales multiplicado por el sesgo total entre la precipitación estimado por los pluviómetros y los valores de satélite, así asumen un sesgo uniforme sobre el espacial.

$$
B=\frac{\sum_{j=1}^{N} Z G(x j)}{\sum_{j=1}^{N} Z S(x j)}
$$

Donde $\mathrm{N}$ es el número de pluviómetros disponibles dentro del dominio del satélite, $Z G(x j)$ and $Z S(x j)$ son los valores de precipitaciones diarias de pluviómetros y satélite correspondiente al pluviómetro localizado $j$.

Double Kernel Smoothing (DS), técnica no paramétrica fue desarrollada por (Li \& Shao, 2010) para aplicaciones con escasez de datos. Este método es la interpolación de los puntos residuales, usando la función residual de kernel y el campo ajustado del satélite por sus residuales.

$$
Z_{D S k}=Z_{S k}-\varepsilon_{D S k}
$$

Residual Inverse Distance Weigthing (RIDW), esta función calcula el sesgo residual en cada par de puntos de píxeles e interpola estos en el campo de satélite usando la ponderación de distancia inversa (IDW) en cada paso de tiempo. Estos residuos se interpolan utilizando la ponderación de la distancia inversa (IDW) y la superficie residual interpolada agrega de nuevo a las estimaciones por satélite (Dinku et al. 2013).

$$
r_{i}=Z_{G, i}-Z_{S, i}
$$

Donde: $Z_{G, i}$ es la observación de precipitación del pluviómetro en el pluviómetro i, $Z_{S, i}$ es la estimación satelital correspondiente y $r_{i}$ es la diferencia residual en el pluviómetro i.

\subsection{Modelación Hidrológica}

La modelación se realizó en base a los datos, corregidos y validados, desarrollados en HEC-DSS de los registros de 10 estaciones automáticas a escala temporal horaria del evento 1.

El evento inició el 29 de marzo de 2014 a las 12:00 y finalizó el 31 de marzo de 2014 a las 12:00, registrándose el caudal pico el 30 de marzo de 2014 a las 15:00 en la estación hidrológica H0472 ubicado en el poblado de Puerto Inca. La precipitación se registró en la cuenca baja y media del área de estudio misma que se pueden observar en la Figura 4. Para la asignación de valor inicial en la modelación hidrológica se consideraron los métodos para procesos hidrológicos de la Figura 6. En el modelo meteorológico se consideró el valor de la precipitación en función del área en las 34 subcuencas, el modelo de subcuenca se consideró los parámetros generados a partir de los métodos de pérdida seleccionado y de los datos generados a partir de información grid y temática, para el caudal base se consideró a partir del hidrograma observado y el tiempo de control se consideró en base al evento 1 .

El modelo hidrológico aplicado en el estudio es el modelo HEC-HMS, semi-distribuido, es decir que las características morfológicas y climatológicas son consideradas como uniformes para cada una de las 34 subcuencas generadas.

La modelación hidrológica de la cuenca hidrográfica considera los siguientes aspectos:

- La información hidrometeorológica registrada por las estaciones automáticas que forman parte de la red instalada en la cuenca del río Cañar es horaria.

- La información satelital de IMERG V03 se muestra a nivel horario y TMPA V07 a nivel 3 horas.

- La información satelital de IMERG V03 y TMPA V07 fue corregida por metodologías no paramétricas de corrección de precipitación satelital MBC, DS y RIWD.

La calibración del modelo hidrológico está en función de un evento de crecida ocurrido el 30 de marzo del 2014.

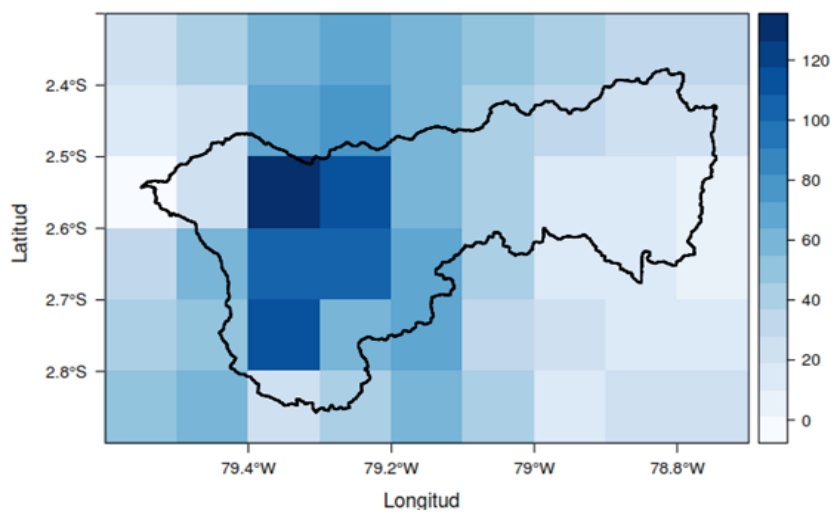

Figura 4. Distribución espacial de la precipitación registrada en el evento1 a $0.1^{\circ} \times 0.1^{\circ}$ 1hora

\subsection{Modelo de Cuenca Cañar}

La definición del modelo de cuenca, se lo realiza a partir de los siguientes criterios: un Modelo Digital de Elevación (DEM por sus siglas en inglés) de 30 metros disponible de la misión Shuttle Radar Topography Mission (SRTM), del tamaño de pixeles de los productos IMERG y TRMM $(10 \mathrm{~km}$ y $25 \mathrm{~km}$, respectivamente) y una distribución espacial adecuada de variables como: precipitación de los productos IMERG y TRMM, número curva $(\mathrm{CN})$ y pendiente. Con la ayuda de la herramienta HEC-GeoHMS, disponible para todas las 
versiones del sistema de información geográfica ArcGIS. El modelo de cuenca generado por la extensión HEC-GeoHMS, cuenta con las características físicas para los componentes de Subcuenca, Unión, Sumidero, mismas que se observan en la Figura 5.

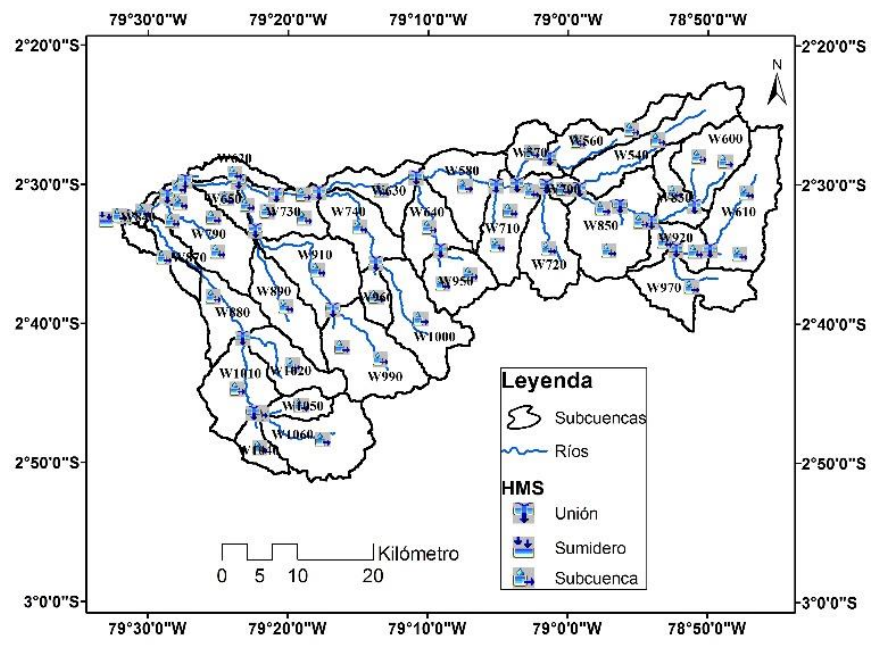

Figura 5. Modelo de Cuenca Hec-Hms

\subsection{Selección de métodos para procesos hidrológicos}

Los métodos a aplicar fueron seleccionados en base a una revisión bibliográfica, los que comúnmente son aplicados en hidrología y han dado buenos resultados (Yang et al. 2017; Shahid et al. 2016). En la Figura 6 muestra los principales parámetros de los métodos de simulación en procesos hidrológicos.

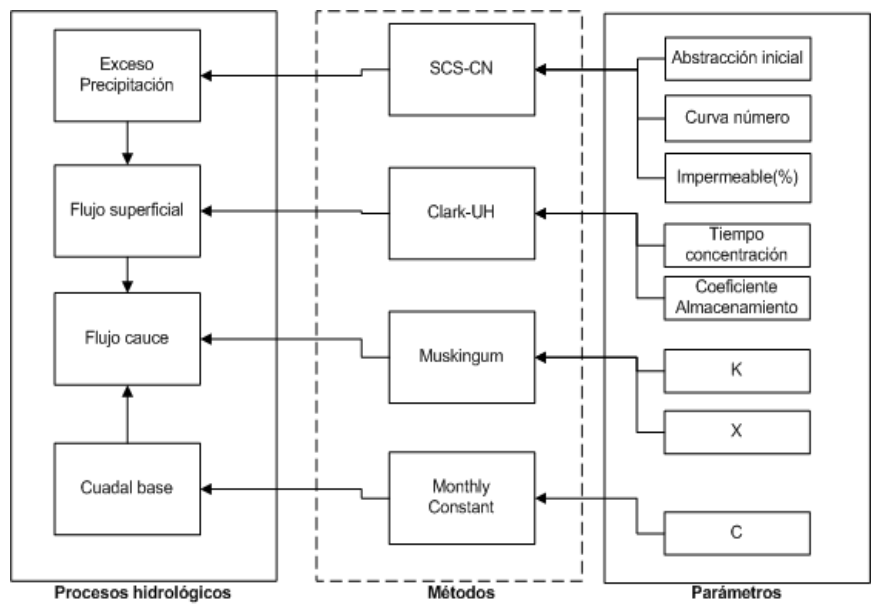

Figura 6. Parámetros de los métodos de simulación en procesos hidrológicos

Los métodos a utilizarse son: en "pérdidas” SCS Número de Curva, al ser uno de los métodos más comunes y además han demostrado buenos resultados (Feldman et al. 2000; Yang et al. 2017); "método de transformación" se utilizará el hidrograma unitario de Clark (Arteaga, 2017; Yang et al. 2017; Shahid et al. 2016) con mejores resultados en relación a otros métodos para su aplicación. En "método de flujo base" en este estudio se utilizó constante, que considera el caudal base constante y puede variar para cada evento.
Los valores iniciales se realizaron para $\mathrm{CN}$ en función del $\mathrm{La}$ asignación inicial de valores a los parámetros se realizó de la siguiente manera. Los valores de Curva Número $(\mathrm{CN})$ se asignaron en función del grupo hidrológico, uso, cobertura, textura y condiciones hidrológicas del suelo; para lo cual se realizó una intersección entre las capas de textura con la cobertura y uso de suelos. Este producto en conjunto con el grupo hidrológico de suelo y condiciones hidrológicas según la clasificación establecida por la Natural Resources Conservation Service (NRCS) define los valores de CN distribuidos en la cuenca. A fin de obtener los valores de $\mathrm{CN}$ para cada una de las 34 subcuencas, se ponderó los valores de $\mathrm{CN}$ en función de los pixeles de valores de $\mathrm{CN}$ en cada subcuenca (Figura 7). Para el hidrograma unitario Clark lluvia - escorrentía, los dos parámetros a ingresar en el modelo son el tiempo de concentración (Tc), el coeficiente de almacenamiento (R), mismos que fueron calculados en base a características fisiográficas de la cuenca. El tránsito del hidrograma por método de Muskingum el coeficiente $\mathrm{k}$ se estimó entre el intervalo de tiempo entre los centros geométricos de los hidrogramas aguas arriba y aguas abajo y para el coeficiente $\mathrm{X}$ de atenuación del hidrograma se consideró el valor de 0.2. En "método de flujo base" en este estudio se utilizó constante, que considera el caudal base constante y que fue determinado como el caudal inmediatamente anterior al inicio del hidrograma de crecida para cada evento.

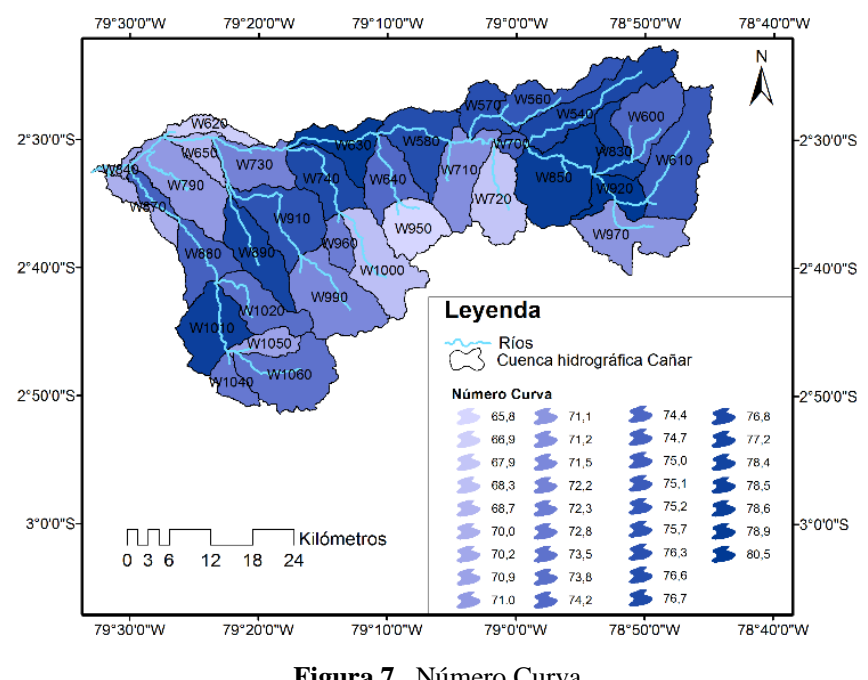

\subsection{Calibración del modelo}

El objetivo de la calibración es optimizar la estructura del modelo y estimar en cada uno de los parámetros de las ecuaciones del modelo. La calibración se realiza ajustando los caudales simulados a caudales observados mediante la optimización de los parámetros en base a criterios seleccionados y funciones objetivo de optimización. (Araghinejad et al. 2013). Los parámetros considerados para la calibración son $\mathrm{CN}, \mathrm{K}$ y $\mathrm{X}$. Los parámetros fueron seleccionados luego de un análisis de sensibilidad de parámetros. 
La calibración se realiza sobre el evento1 (29/03/201431/03/2014) la identificación de parámetros se realiza manualmente.

La evaluación gráfica se realizó comparando visualmente los hidrogramas simulado y observado, analizando las funciones objetivo como diferencia caudal pico, diferencia tiempo del pico y diferencia volumen de escorrentía. Para la evaluación estadística se utilizará un criterio que permita establecer la precisión o error del modelo. En este estudio al tratarse de un evento puntual se consideró para el análisis estadístico de precisión del modelo al Porcentaje de Sesgo (PBIAS) y el Nash- Sutcliffe (NSE) (Yang et al. 2017).

El PBIAS mide la tendencia en cada paso de tiempo a ser mayor o menor los resultados de los modelos simulados con respecto a los caudales observados. El valor óptimo de PBIAS es cero, con valores de baja magnitud indica una simulación precisa del modelo. Los valores positivos indican sesgo de subestimación del modelo y valores negativos indica sesgo de sobreestimación del modelo (Moriasi et al. 2007).

La eficiencia Nash-Sutcliffe (NSE) mide la magnitud relativa de la varianza residual o variabilidad de los datos observados comparada con la varianza de los datos simulados (Nash \& Sutcliffe, 1970).

$$
N S E=1-\left[\frac{\sum_{i=1}^{n}\left(Y_{i}^{o b s}-Y_{i}^{s i m}\right)^{2}}{\sum_{i=1}^{n}\left(Y_{i}^{o b s}-Y^{m e a n}\right)^{2}}\right]
$$

Donde: $Y_{i}^{o b s}$ es el i-ésimo valor observado, $Y_{i}^{\text {sim }}$ es el i-ésimo valor simulado, $Y^{\text {mean }}$ es el promedio de datos observados y $\mathrm{n}$ es el total de número de observaciones. El valor de los rangos del NSE - $\infty$ y 1 . Donde NSE de valor 1 es el óptimo, los valores entre 0 y 1 son considerados como aceptables de desempeño, mientras que valor $\leq 0$ indica que la media del valor observado es mejor predictor que el valor simulado el cual indica un valor insatisfactorio.

Los criterios de evaluación para PBIAS y NSE, según Moriasi et al. (2007), se los asigna en función de los valores contenidos en la Tabla 3.

Tabla 3. Criterios estadísticos para la evaluación de desempeño del modelo

\begin{tabular}{ccc}
\hline CRITERIO & VALOR & CALIFICACIÓN \\
\hline \multirow{3}{*}{ PBIAS } & PBIAS $< \pm 10$ & Muy Bueno \\
& $\pm 10 \leq \mathrm{PBIAS} \pm 15$ & Bueno \\
& $\pm 15 \leq \mathrm{PBIAS} \pm 25$ & Satisfactorio \\
& PBIAS \pm 25 & Insatisfactorio \\
& $0,75<\mathrm{NSE} \leq 1,00$ & Muy Bueno \\
NSE & $0,65<\mathrm{NSE} \leq 0,75$ & Bueno \\
& $0,50<\mathrm{NSE} \leq 0,65$ & Satisfactorio \\
& NSE $\leq 0,50$ & Insatisfactorio \\
\hline
\end{tabular}

Para la evaluación de los hidrogramas simulado y observado, se analizará las funciones objetivo como diferencia caudal pico, diferencia tiempo del pico y diferencia volumen de escorrentía.

\subsection{Sensibilidad de parámetros del modelo}

Este análisis permite definir la importancia de cada parámetro. Además entender cómo funciona el modelo o las razones por las que no funciona correctamente, aprender de su funcionamiento y detectar si el modelo está sobreparametrizando, es decir si existen parámetros a los que el modelo resulta insensible. Para este análisis se ejecuta el modelo variando un parámetro específico y los demás parámetros se mantienen fijos. Sin embargo la sensibilidad a un parámetro dependerá de los valores adoptados por los demás parámetros, con lo que puede ser complejo realizar un análisis de sensibilidad.

Para el presente estudio el análisis de sensibilidad se realizó a la par con las pruebas de calibración ya que al variar cada uno de los parámetros del modelo para encontrar el óptimo, se identificará que parámetro tiene mayor influencia en resultados del modelo, tomando como base los parámetros determinados por (Arteaga, 2017).

\subsection{Validación del modelo}

Se realiza con el fin de verificar la calidad de los ajustes de los parámetros obtenidos en la mejor prueba de calibración, que serán reemplazados en los eventos a analizarse.

Se evalúa el modelo para asegurar que cumple con los objetivos para los cuales fue desarrollado. Esta evaluación consiste en validar primero el modelo probando la integridad de su estructura y los conjuntos de parámetros obtenidos durante su calibración. La validación del modelo no es equivalente a la verificación del modelo. El objetivo de la verificación es comprobar que el modelo es "realista". Es decir que proporciona una representación lo más cercana posible a la realidad (Hingray et al. 2015).

\section{RESULTADOS Y DISCUSIÓN}

\subsection{Evaluación de productos satelitales en base a observaciones.}

\section{Indicadores cuantitativos}

El coeficiente de correlación Figura 8, de las estimaciones con los valores observados para los eventos registrados. Los datos de estimación que mejor coeficientes de correlación positiva registra es IMERG V03 a escala temporal $1 \mathrm{~h}$ especialmente para los eventos $1,4,7$ y 8 siendo el evento 1 el mejor CC. Mientras que los datos TMPA V7 registran un coeficiente de correlación bajo de relación positiva o negativa para alguno de sus cuartiles. Sin embargo para los eventos 2, 3, 5 y 6 TMPA V7 registran un mejor coeficiente de correlación que IMERG V03.

Se debe destacar que en los eventos 1,4 y 5 la relación en general es directa entre los productos satelitales y valores observados. Lo que indica alto valores de precipitación observada corresponden altos valores de intensidad de precipitación de los productos IMERG y TMPA. Varios factores pueden contribuir al bajo $\mathrm{CC}$ tanto en IMERG Y 
TMPA siendo entre estos la topografía y clima complejo, el escaso número de pluviómetros que no registran adecuadamente la precipitación.

El sesgo relativo Figura 9, para las estimaciones con los valores observados en los eventos registrados. Para IMERG V03 registra sesgos positivos y negativos en sus cuartiles, pero en su mayoría de cuartiles son positivos para los eventos 1, 2, $3,4,5,6$, y 8 dando lugar a una mejor estimación de registro de precipitación satelital en referencia a estimación de TMPA. Mientras que TMPA V7 registra sesgo positivo y negativo en sus cuartiles, pero en su mayoría de cuartiles son negativos para los eventos indicando un menor registro de precipitación satelital. El evento 5 registra el menor sesgo tanto para IMERG V03 y TMPA V7.

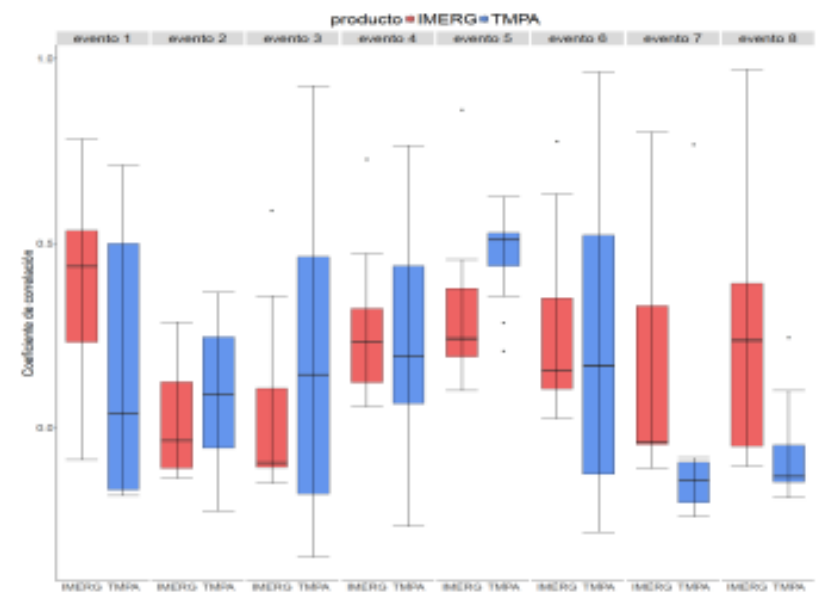

Figura 8. Coeficientes de correlación

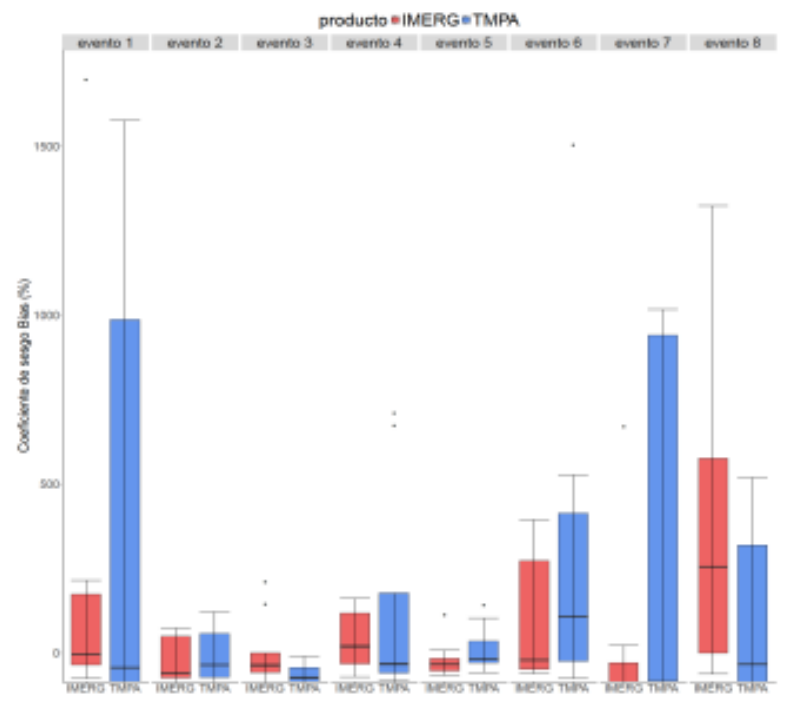

Figura 9. Coeficientes de sesgo

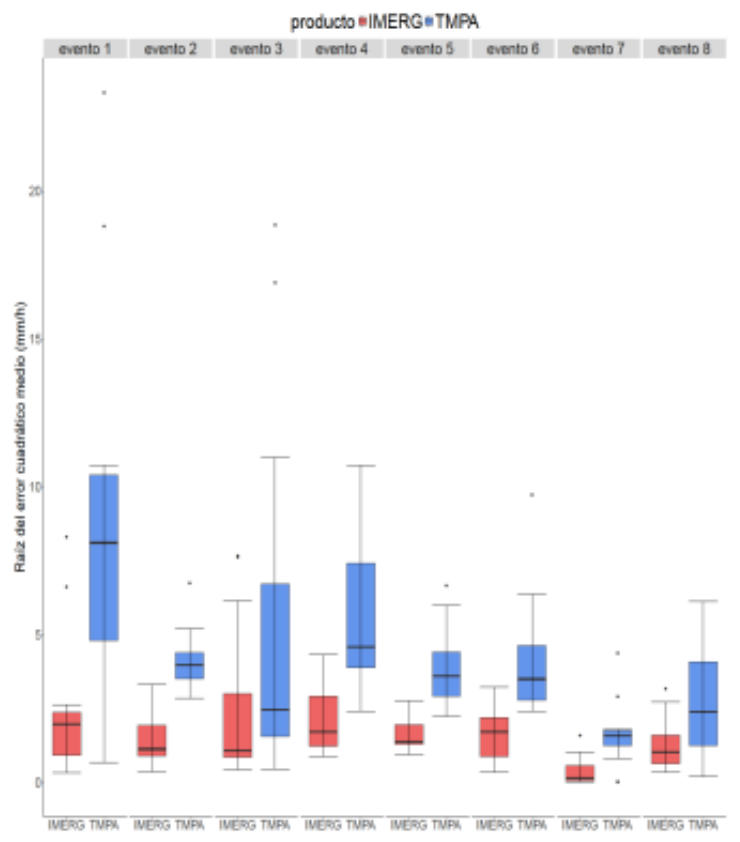

Figura 10. Raíz del error cuadrático medio producto =IMERG $=$ TMPA

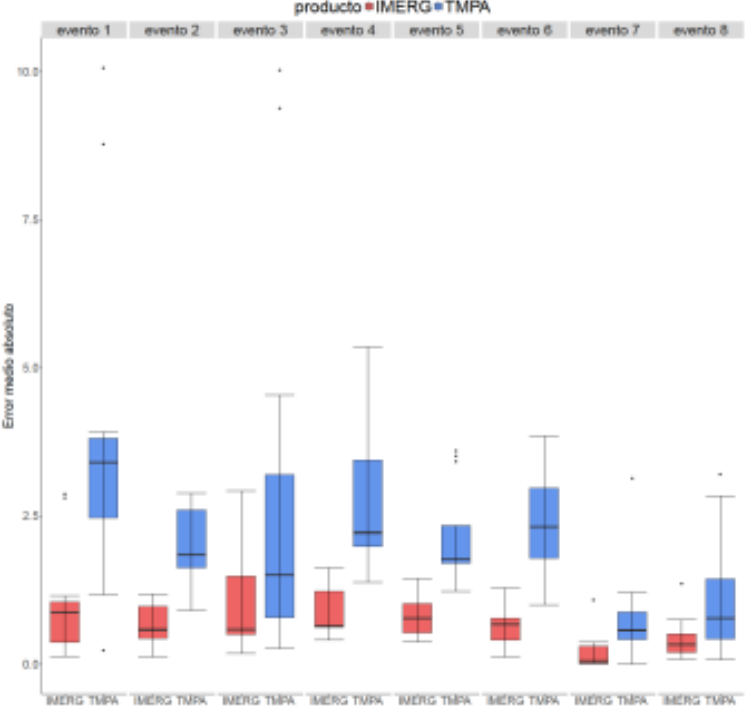

Figura 11. Error medio absoluto

El PBIAS positivo en el TMPA sobrestima las observaciones de precipitación mientras que para IMERG se reduce sustancialmente, reducción que es corroborado por Manz et al. (2017) para la subregiones PCN y AW en la que se encuentra inmersa la cuenca en estudio.

La raíz del error medio cuadrático Figura 10, en las estimaciones con los valores observados para los eventos registrados, IMERG V03 es la que registra menor error para los eventos 1, 2, 3, 4, 5, 6,7 y 8, mientras que TMPA V7 registra mayores errores y valores atípicos para todos los eventos.

Los valores RMSE muestran una reducción consistente para IMERG en comparación con TMPA, reducción que es corroborado por Manz et al. (2017) para todas las subregiones en la que está incluida el estudio. 
El error medio absoluto Figura 11, en las estimaciones con los valores observados en los eventos registrados, IMERG V03 es la que registra menor error para todos los eventos $1,2,3,4$, 5, 6,7 y 8 mientras que TMPA V7 registra mayor error para todos los eventos. Los valores MAE muestran una reducción consistente para IMERG en comparación con TMPA.

\section{Indicadores cualitativos}

Se ha obtenido las siguientes medidas de evaluación de productos satelitales:

- Probabilidad de detección $(P O D)$

- Falsa alarma (FAR)

- CDF RATIO

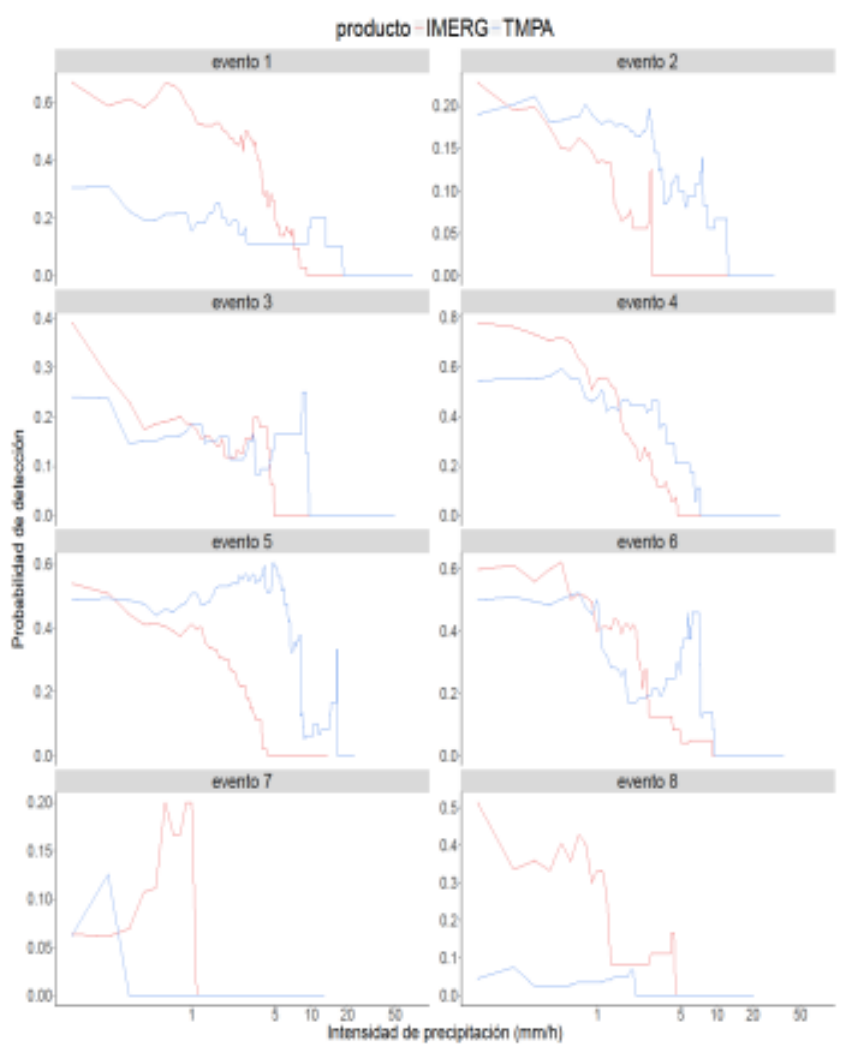

Figura 12. Probabilidad de detección

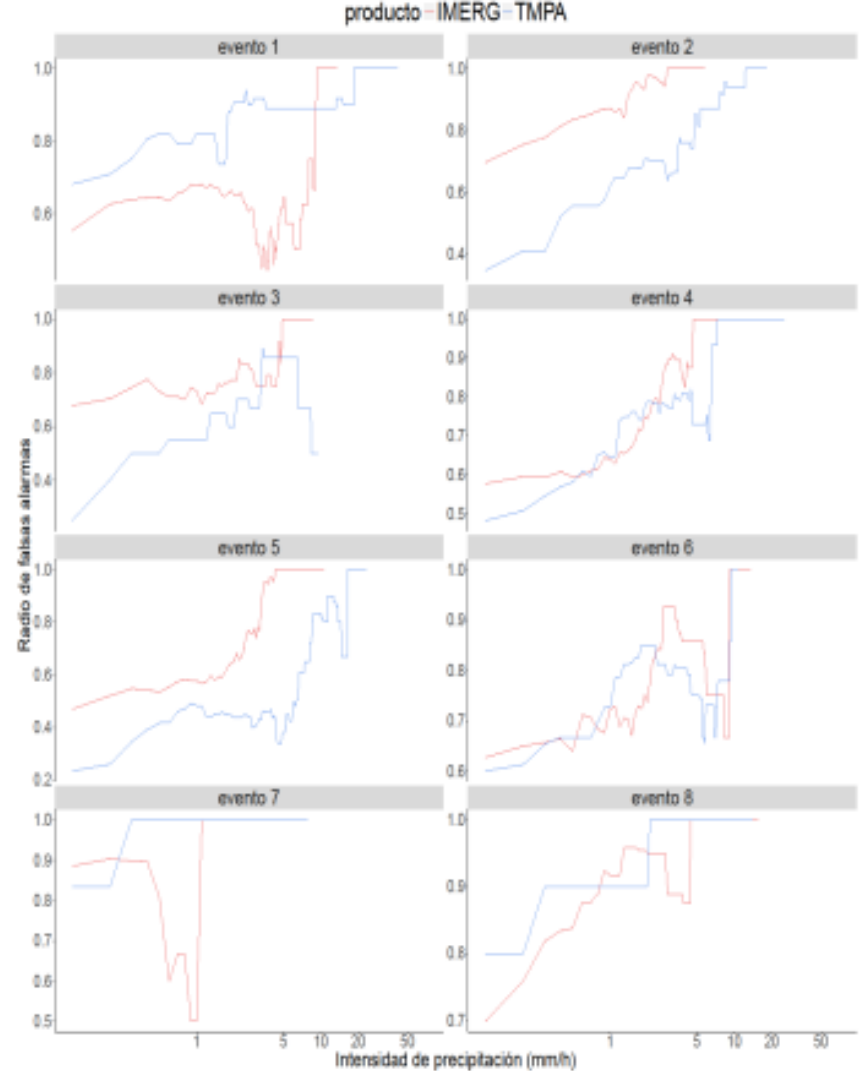

Figura 13. Radio de falsas alarmas

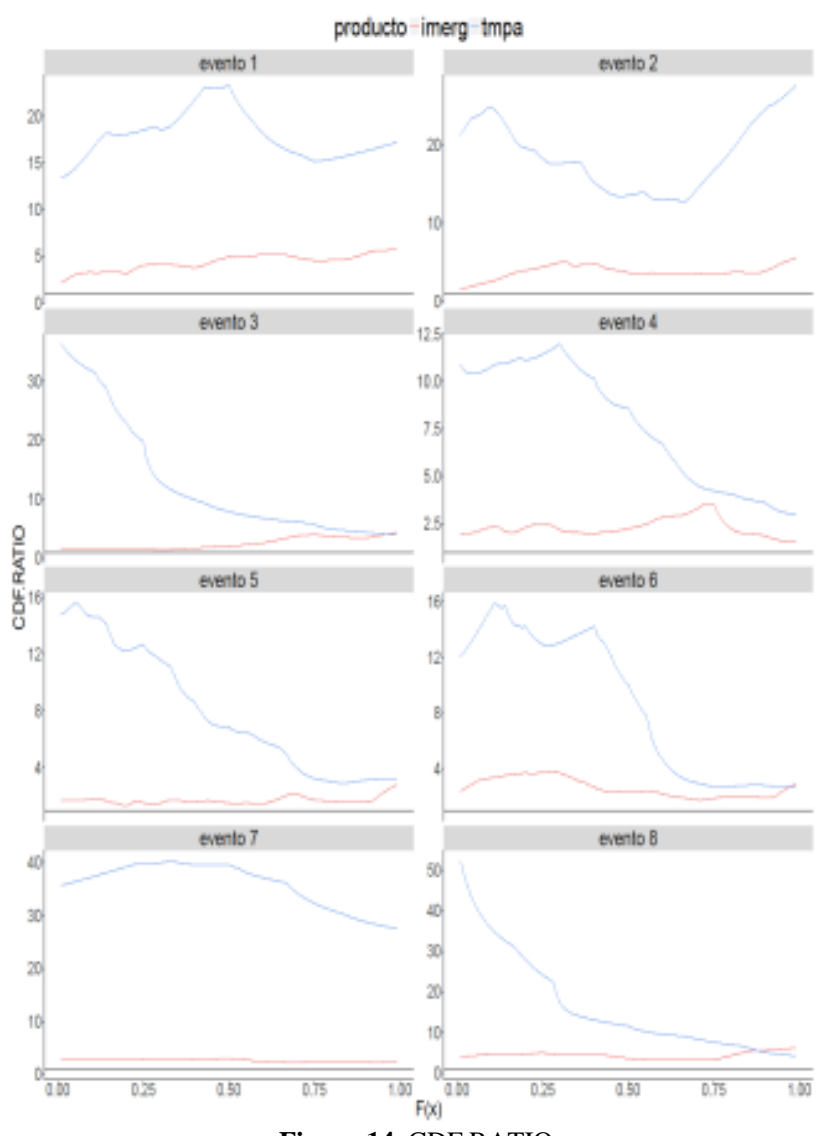

Figura 14. CDF RATIO

La probabilidad de detección Figura 12, para las estimaciones con los valores observados en los eventos evaluados, IMERG 
V03 registra mejor probabilidad de detección para eventos 1 , $3,4,6,7$ y 8 especialmente a intensidades bajas y estas disminuyen a medida que aumenta la intensidad. Para TMPA V7 para los eventos 2 y 5 registra mejor probabilidad de detección, mientras que para IMERG y TMPA el evento 7 registra menor POD. La reducción del POD del satélite se incrementa conforme aumenta la intensidad de precipitación, resultado que es corroborado por Manz et al. (2017); Tang et al. 2016 \& Córdova, (2017).

El radio de falsas alarmas Figura 13, en las estimaciones con los valores observados de los eventos registrados, IMERG V03 para los eventos 2, 3, 4, 5 y 6 y TMPA V7 para los eventos 1,7 y 8 registran más falsas alarmas a intensidades bajas y que se incrementan con una mayor intensidad de precipitación, resultado que corrobora lo reportado por Manz et al. (2017); Andrade, (2016). En general de acuerdo a los resultados IMERG presenta más falsa detección de precipitaciones que en realidad no sucedieron que TMPA, resultado difiere del registrado para las subregiones PCN y AW por Manz et al. (2017).

El CDF RATIO Figura 14, en las estimaciones con los valores observados de los eventos registrados, en todos los eventos se registran una sobrestimación en la intensidad de precipitación tanto para productos IMERG V03 y TMPA V7. Además IMERG V03 para eventos 1, 2, 3, 4, 5, 6,7 y 8 registra un menor CDF RATIO que TMPA V7. Sin embrago para el evento 8 TMPA V7 registra un CDF RATIO más bajos en el percentil 100. En general los resultados muestran que IMERG presenta un CDF RATIO más cercano a uno que TMPA resultados que ratifica lo indicado por Manz et al. (2017).

\section{Métodos de fusión de datos de estimación de precipitación.}

Con la modelación hidrológica se puedo observar las estimaciones de caudal a partir de datos de precipitación con los métodos de fusión DS, RIDW y MBC. El método de DS es el que mejor resultado registra seguido por RIDW. Resultado que es validado por Nerini et al., (2015) cuyo mejor resultado, para cuencas con escasez de datos, fue DS en evaluación hidrológica.

\section{Modelación Hidrológica}

La calibración se realizó sobre el evento1 (29/03/2017$31 / 03 / 2014$ ), los parámetros obtenidos en la mejor prueba de calibración del evento1, fueron reemplazados en los ocho eventos de crecidas restantes, para analizar si dichos parámetros pueden ser generalizados para los eventos en cuestión.

\subsection{Simulación evento 1 en base a observaciones de estaciones automáticas}

En la Tabla 4, se muestran los resultados de simulación bajo los parámetros iniciales establecidos por el método de transformación H.U Clark a escala espacio temporal 1 hora y 3 horas.

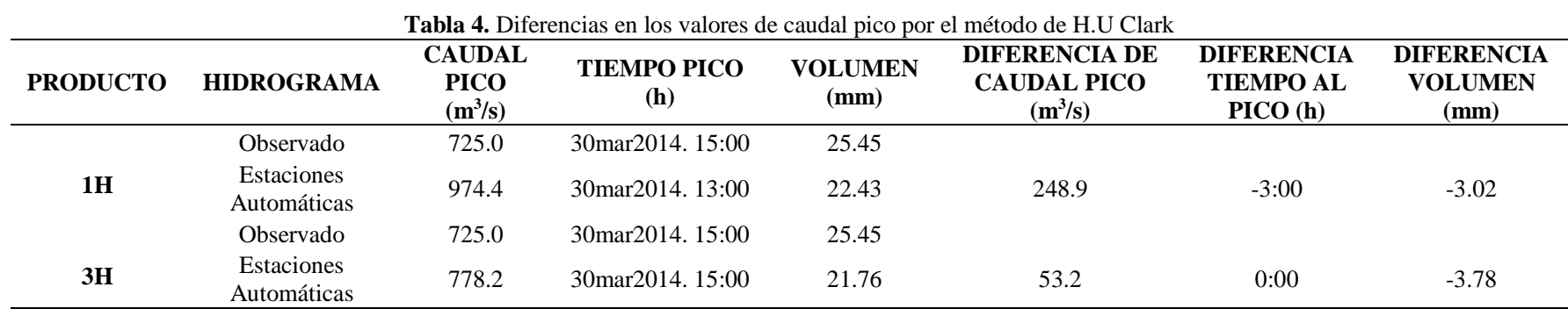

\subsection{Calibración}

En el análisis de sensibilidad para cada uno de los métodos de transformación, H.U Snyder, H.U SCS y H.U Clark (Arteaga 2017) indica que el método H.U Clark es el que mejor se ajusta, al hidrograma generado por los valores observados en la estación H0472 Puerto Inca, por tanto se procede a realizar la calibración del modelo considerando esos resultados.

óptimos de los parámetros calibrados para productos a 1 hora se consideró el valor de $\mathrm{CN}$ en una reducción en un $5 \%$ del valor inicial de igual manera se realizó un incremento en $20 \%$ en el parámetro k de Muskingum. Para productos agregados a 3 horas se consideró el valor de $\mathrm{CN}$ en una reducción en un $2 \%$ del valor inicial y el parámetro $\mathrm{k}$ de Muskingum se mantuvo en su valor inicial.

Para la fase de calibración del modelo a partir del valor inicial, los parámetros considerados fueron del modelo de subcuenca el CN, y del modelo de Muskingum K y X. Para el CN el producto de las capas textura y cobertura del suelo, con el grupo hidrológico de suelo y condiciones hidrológicas según la clasificación establecida por la NRCS, para CN a cada una de las 34 subcuencas, se ponderó los valores de $\mathrm{CN}$ en función del área del raster de valores de $\mathrm{CN}$. El coeficiente k se estimó entre el intervalo de tiempo entre los centros geométricos de los hidrogrmas aguas arriba y aguas abajo y para el coeficiente $\mathrm{X}$ de atenuación del hidrograma se consideró el valor de 0.2 . De los resultados simulados, el mejor desempeño y los valores 


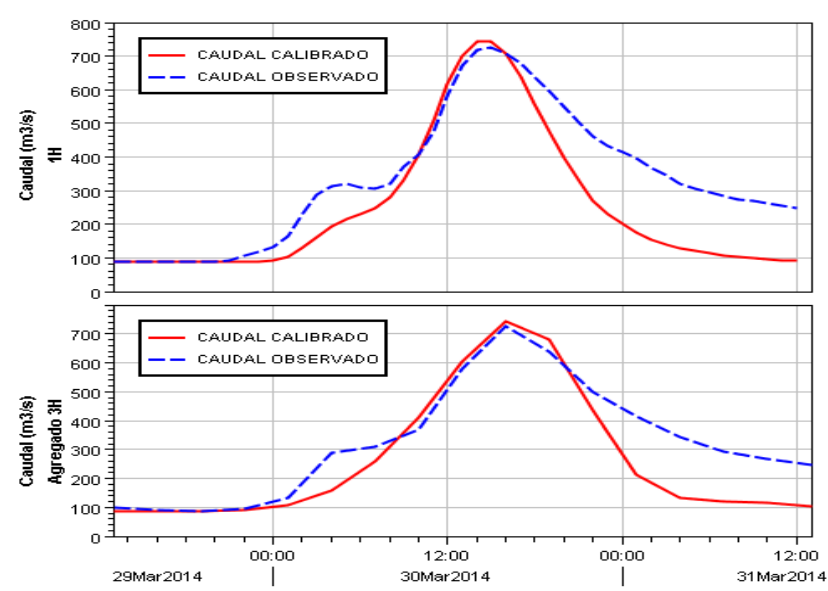

Figura 15. Simulación bajo parámetros de calibración

En la figura 15, los resultados de las simulaciones a partir de los parámetros de calibración para productos a 1 hora se registran un caudal de $744.9 \mathrm{~m}^{3} / \mathrm{s}$ observándose una diferencia $19.9 \mathrm{~m}^{3} / \mathrm{s}$ con el caudal observado. Para productos agregados a 3 horas se registra un caudal de $744.8 \mathrm{~m}^{3} / \mathrm{s}$ observándose una diferencia de $19.8 \mathrm{~m}^{3} / \mathrm{s}$ con el caudal observado.

3.4 Simulación eventol para métodos de fusión

En la Figura 16, para simulaciones con el modelo calibrado se observa que los caudales generados a partir de productos satelitales IMERG V03, TMPA V7 y métodos de corrección estos registran una subestimación en los caudales. Además se registra un desfase en el tiempo al pico tanto en IMERG V03 y TMPA V7 siendo mayor en IMERG.

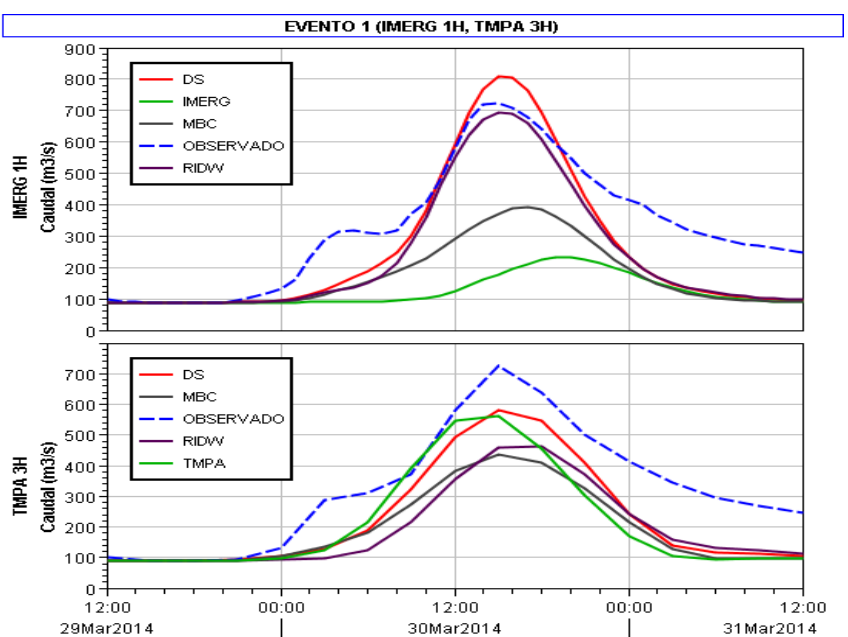

Figura 16. Caudales evento 1 IMERG V03 y TMPA V7

\subsection{Re calibración a partir del evento 1}

En este estudio se destaca la necesidad de mejorar la recuperación de la precipitación por satélite para permitir un uso más apropiado de los productos de precipitación por satélite para las aplicaciones de eventos de inundaciones.

Considerando la observación anterior y con el fin de recalibrar el modelo a partir de información de grillas tanto para IMERG V03 y TMPA V7 a escala espacio temporal 1hora y 3 horas respectivamente, se realizó la misma considerando tanto el ajuste gráfico del hidrograma y criterio PBIAS. Para el producto IMERG se recalibró a partir del método de corrección RIDW y para el producto TMPA con el método de corrección DS.

Tabla 5. Valores de caudal métodos de corrección en IMERG V03 recalibrado evento1.

\begin{tabular}{|c|c|c|c|c|c|c|}
\hline HIDROGRAMA & $\begin{array}{c}\text { CAUDAL PICO } \\
\left(\mathbf{m}^{3} / \mathbf{s}\right)\end{array}$ & $\begin{array}{l}\text { TIEMPO AL } \\
\text { PICO } \\
\text { (h) } \\
\end{array}$ & $\begin{array}{c}\text { VOLÚMEN } \\
(\mathrm{mm})\end{array}$ & $\begin{array}{c}\text { DIFERENCIA DE } \\
\text { CAUDAL PICO } \\
\left(\mathbf{m}^{3} / \mathbf{s}\right)\end{array}$ & $\begin{array}{c}\text { DIFERENCIA } \\
\text { TIEMPO AL } \\
\text { PICO (h) } \\
\end{array}$ & $\begin{array}{c}\text { DIFERENCIA } \\
\text { VOLÚMEN } \\
(\mathrm{mm})\end{array}$ \\
\hline OBSERVADO & 725.0 & 30mar2014. 15:00 & 25.73 & & & \\
\hline DS & 857.3 & 30mar2014. 15:00 & 22.01 & 132.3 & 0 & -3.71 \\
\hline RIDW & 744.8 & 30mar2014. 15:00 & 20.36 & 19.8 & 0 & -5.37 \\
\hline MBC & 425.8 & 30mar2014. 17:00 & 14.97 & -299.2 & 2 & -10.76 \\
\hline IMERG & 255.6 & 30mar2014. 20:00 & 10.8 & -469.4 & 5 & -14.93 \\
\hline $\begin{array}{c}\text { ESTACIONES } \\
\text { AUTOMÀTICAS }\end{array}$ & 744.9 & 30mar2014. 15:00 & 19.07 & 19.9 & 0 & -6.66 \\
\hline
\end{tabular}

En la Tabla 5, Tabla 6 y Figura 17, se muestra el análisis de caudal recalibrado del evento1, para los productos IMERG V03, TMPA V7, los métodos de corrección y estaciones automáticas. Para los caudales generados a partir de productos IMERG V03 registran una sobrestimación para DS y RIDW, mientras que en los caudales generados a partir de TMPA V7 se registra una subestimación en los caudales. De igual manera se registra un desfase en el tiempo al pico tanto en productos IMERG y TMPA siendo mayor para IMERG. Los hidrogramas se ajustan bien al hidrograma observado, mismo que se indica en el criterio de PBIAS y NSE. Estos resultados se debe seguramente a que el registro de precipitación en las estaciones pluviométricas fue ajustado por tanto los métodos de corrección se ajustaron muy bien. Además cabe indicar que los mejores resultados para DS y RIDW obtenidos para este evento son significativamente mejores a los indicados por Arteaga, (2017) en su estudio y esto seguramente porque los productos satelitales mejoran la distribución espacial registrada con los pluviómetros considerando los métodos de corrección. 
Tabla 6. Valores de caudal métodos de corrección en TMPA V7 recalibrado evento1.

\begin{tabular}{|c|c|c|c|c|c|c|}
\hline HIDROGRAMA & $\begin{array}{l}\text { CAUDAL PICO } \\
\qquad\left(\mathrm{m}^{3} / \mathrm{s}\right)\end{array}$ & $\begin{array}{c}\text { TIEMPO AL } \\
\text { PICO } \\
\text { (h) } \\
\end{array}$ & $\begin{array}{l}\text { VOLÚMEN } \\
\quad(\mathbf{m m})\end{array}$ & $\begin{array}{c}\text { DIFERENCIA DE } \\
\text { CAUDAL PICO } \\
\left(\mathrm{m}^{3} / \mathrm{s}\right)\end{array}$ & $\begin{array}{c}\text { DIFERENCIA } \\
\text { TIEMPO AL } \\
\text { PICO (h) } \\
\end{array}$ & $\begin{array}{c}\text { DIFERENCIA } \\
\text { VOLÚMEN } \\
(\mathbf{m m}) \\
\end{array}$ \\
\hline OBSERVADO & 709.0 & 30mar2014. 16:00 & 24.69 & \multirow[b]{2}{*}{-109.6} & & \\
\hline RIDW & 599.4 & 30 mar2014. 16:00 & 17.86 & & 0 & -6.83 \\
\hline DS & 711.2 & 30 mar2014. 16:00 & 20.82 & 2.2 & 0 & 3.87 \\
\hline MBC & 527.5 & 30mar2014. 16:00 & 17.67 & -181.5 & 0 & -7.02 \\
\hline TMPA & 710.4 & 30mar2014. 13:00 & 19.78 & 1.4 & -3 & -4.91 \\
\hline $\begin{array}{c}\text { ESTACIONES } \\
\text { AUTOMÀTICAS }\end{array}$ & 744.9 & 30 mar2014. 16:00 & 20.88 & 35.9 & 0 & -3.81 \\
\hline
\end{tabular}

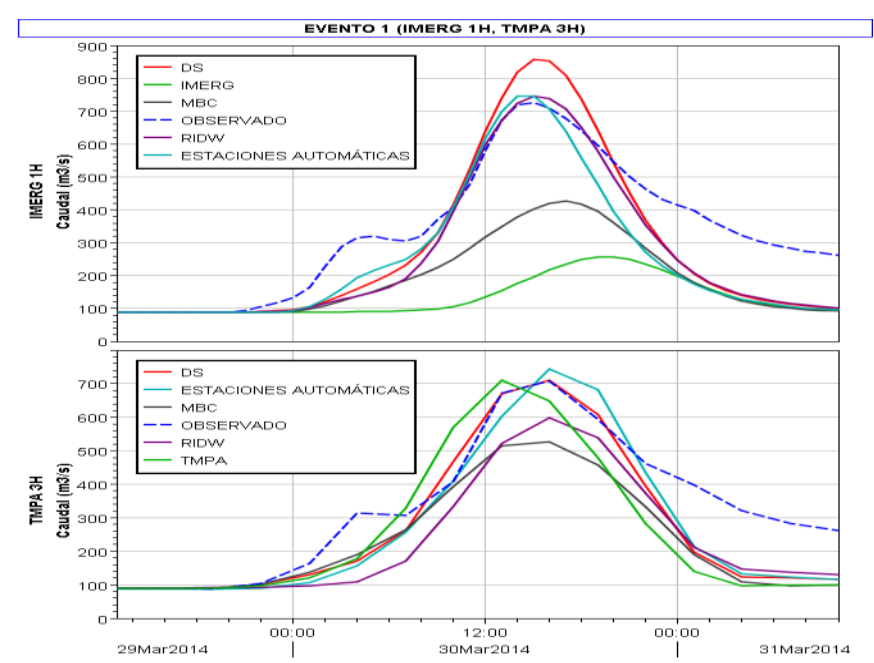

Figura 17. Caudales recalibrados evento 1 IMERG V03 y TMPA V7

\section{Validación}

La validación consistió en utilizar los modelos recalibrados a partir de información satelital del evento 1, tanto para IMERG V03 y TMPA V7 y aplicar estos valores en los ocho eventos evaluados. A continuación se presenta el evento en el que el modelo proporciona una representación más ajustada y razonable de esta realidad, adaptada al objetivo buscado (evento 5).

En la Tabla 7, Tabla 8, Figura 18, se registra que los productos de precipitación satelital de IMERG son más ajustados y por tanto también los métodos de corrección evaluados. Para IMERG el RIDW presenta una diferencia en caudal de $2.9 \mathrm{~m}^{3} / \mathrm{s}$, tiempo al pico $2 \mathrm{~h}$, volumen $-2.91 \mathrm{~mm}$ y un PBIAS de 0.79 y NSE de 0.99 de calificación muy bueno, seguido por el método de corrección DS. Para productos TMPA se registra un subestimación de precipitación sin embargo con los métodos de corrección el mejor ajuste se dio para RIDW con una diferencia menor en caudal de $-23.3 \mathrm{~m}^{3} / \mathrm{s}$, tiempo al pico Oh, volumen $-4.48 \mathrm{~mm}$, un PBIAS de 0.79 y NSE de 0.99 de calificación muy bueno. Siendo resultados más significativos a los reportados por Arteaga, (2017) para este mismo evento. Además los resultados muy buenos para los criterio PBIAS y NSE, para los hidrogramas por los métodos de corrección, se debe seguramente al registro muy bueno de precipitación por las estaciones pluviométricas para el evento.

\begin{tabular}{|c|c|c|c|c|c|c|}
\hline HIDROGRAMA & $\begin{array}{l}\text { CAUDAL } \\
\text { PICO } \\
\left(\mathbf{m}^{3} / \mathbf{s}\right) \\
\end{array}$ & $\begin{array}{l}\text { TIEMPO AL } \\
\text { PICO } \\
\text { (h) } \\
\end{array}$ & $\begin{array}{c}\text { VOLÚMEN } \\
(\mathbf{m m})\end{array}$ & $\begin{array}{c}\text { DIFERENCIA DE } \\
\text { CAUDAL PICO } \\
\left(\mathbf{m}^{3} / \mathbf{s}\right)\end{array}$ & $\begin{array}{c}\text { DIFERENCIA } \\
\text { TIEMPO AL } \\
\text { PICO (h) } \\
\end{array}$ & $\begin{array}{c}\text { DIFERENCIA } \\
\text { VOLÚMEN } \\
(\mathbf{m m})\end{array}$ \\
\hline OBSERVADO & 364.8 & 29mar2015. 06:00 & 27.85 & & & \\
\hline RIDW & 361.9 & 29mar2015. 08:00 & 24.94 & -2.9 & 2 & -2.91 \\
\hline DS & 373.0 & 29mar2015. 08:00 & 25.44 & 8.2 & 2 & -2.41 \\
\hline MBC & 349.1 & 29mar2015. 08:00 & 26.67 & -15.7 & 2 & -1.18 \\
\hline IMERG & 170.8 & 29mar2015. 00:00 & 18.4 & -194 & 18 & -9.45 \\
\hline $\begin{array}{c}\text { ESTACIONES } \\
\text { AUTOMÀTICAS }\end{array}$ & 408 & 29mar2015. 05:00 & 24.49 & 43.2 & -1 & -3.36 \\
\hline
\end{tabular}

\begin{tabular}{|c|c|c|c|c|c|c|}
\hline HIDROGRAMA & $\begin{array}{c}\text { CAUDAL } \\
\text { PICO } \\
\left(\mathbf{m}^{3} / \mathbf{s}\right) \\
\end{array}$ & $\begin{array}{l}\text { TIEMPO AL } \\
\text { PICO } \\
\text { (h) } \\
\end{array}$ & $\begin{array}{l}\text { VOLÚMEN } \\
(\mathbf{m m})\end{array}$ & $\begin{array}{c}\text { DIFERENCIA DE } \\
\text { CAUDAL PICO } \\
\left(\mathrm{m}^{3} / \mathrm{s}\right)\end{array}$ & $\begin{array}{c}\text { DIFERENCIA } \\
\text { TIEMPO AL } \\
\text { PICO (h) } \\
\end{array}$ & $\begin{array}{c}\text { DIFERENCIA } \\
\text { VOLÚMEN } \\
(\mathbf{m m}) \\
\end{array}$ \\
\hline OBSERVADO & 363.1 & 29mar2015. 07:00 & 27.42 & & & \\
\hline RIDW & 339.8 & 29mar2015. 07:00 & 22.94 & -23.3 & 0 & -4.48 \\
\hline DS & 411.5 & 29mar2015. 07:00 & 25.69 & 48.4 & 0 & -1.73 \\
\hline TMPA & 270.3 & $29 \operatorname{mar} 2015.10: 00$ & 21.28 & -92.8 & 3 & -6.14 \\
\hline $\begin{array}{l}\text { ESTACIONES } \\
\text { AUTOMÀTICAS }\end{array}$ & 431.2 & 29 mar2015, 07:00 & 26.55 & 68.1 & 0 & -0.87 \\
\hline
\end{tabular}




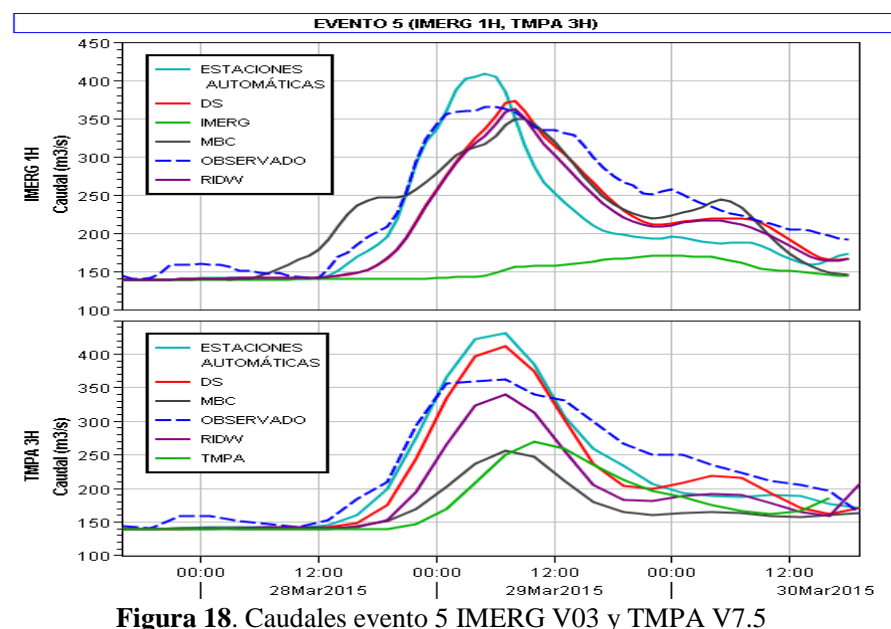

Figura 18. Caudales evento 5 IMERG V03 y TMPA V7.5

\section{CONCLUSIONES}

Este estudio realizó la validación IMERG V03 y TMPA V7 con una red de 13 pluviómetros en la cuenca del río Cañar durante el período de marzo de 2014 a diciembre de 2015. Los productos IMERG V03 y TMPA V7 subestiman las intensidades de precipitación, misma que se atribuye a la topografía fuertemente accidentada que registra POD bajos y que disminuyen al incrementar la intensidad y FAR altos que se incrementan al incrementar la intensidad de precipitación, esta concuerda con lo señalado por Tang et al. (2016).

Durante el período de evaluación IMERG y TMPA a escalas espaciales y temporales de ( $1 \mathrm{~h}$ y $3 \mathrm{~h}$, respectivamente), los resultados muestran que el IMERG tiene una mejor capacidad cuantitativa de estimación de la intensidad de lluvia que TMPA, resultado que corrobora lo registrado por Manz et al., (2017).

En la evaluación de productos satelitales considerando los métodos de fusión, los productos satelitales IMERG y TMPA mejoran la distribución espacial registrada con los pluviómetros considerando los métodos de corrección como Double Kernel Smoothing, Residual Inverse Distance Weigthing. En base a la estación hidrológica de Puerto Inca se realizó la evaluación hidrológica identificándose que el método de corrección DS es el que mejor resultados registra, seguido por RIDW. En esta evaluación el mejor ajuste gráfico y estadístico se registró para el evento 1 y evento 5 por los métodos de corrección y esto se debe a que para estos eventos las estaciones automáticas representan de manera adecuada la precipitación. Además se puede evidenciar al presentar mejor POD, mejor CC y menor MAE.

La diferencia observada en el descenso del hidrograma tanto en el evento 1 como en el 5 se podría originar al haber considerado al caudal base como constante a nivel mensual en estos eventos. Este caudal base se obtuvo en base al caudal previo al inicio de cada evento. A pesar de esta limitación el caudal máximo se aproxima al real, así como el volumen del hidrograma está dentro delos rangos considerados como aceptables
Este estudio también ha resaltado que el modelo hidrológico HEC-HMS calibrado muestra que para productos satelitales IMERG V03 y TMPA V7 fusionados, se ajustan a eventos de crecida en los que los productos satelitales registran una alta probabilidad de detección de lluvia (POD) y de mayor intensidad de precipitación.

Los productos satelitales IMERGV03 y TMPAV7 fusionados con observaciones de campo mediante los métodos DS y RIDW mejoran los resultados de la simulación de caudales de crecidas en comparación si solo se usaran datos de observaciones pluviométricas únicamente. Los resultados nos permiten aportar con evidencia sobre la aplicación de productos de precipitación satelital para simular caudales de crecidas, especialmente para cuencas con escasos datos de campo y para eventos con gran intensidad.

\section{RECOMENDACIONES}

En una evaluación adicional se debe considerar en el modelo hidrológico datos de entrada de los productos IMERG V05 con el fin de comparar si mejoran las estimaciones con respecto de la versión V03. Así mismo, se pueden explorar los productos de precipitación satelital en tiempo casi real (NRT), que servirían a una potencial aplicación en la detección de crecidas en tiempo casi real, especialmente importante para cuencas de gran tamaño. Si bien los resultados presentados IMERG presenta mejores resultados estas también deberían ser evaluados a una escala espacio temporal diaria para estimaciones IMERG V03 y V05 en la estimación de precipitaciones máximas en 24 horas, con el fin de observar su posible uso en la estimación de intensidades máximas y uso en la determinación de hietogramas de diseño y modelación para eventos de crecidas para diferentes periodos de retorno. Adicionalmente se debería considerar probar un modelo hidrológico continuo, a paso de tiempo horario para mejorar la respuesta de la cuenca en cuanto a considerar un posible almacenamiento de agua en el suelo/subsuelo y que se reflejen más adecuadamente los caudales.

\section{AGRADECIMIENTO}

El presente estudio fue posible gracias a la Escuela Politécnica Nacional por intermedio del proyecto de investigación PIJ-1514: "Evaluación de la aplicación potencial de la precipitación satelital obtenida de la misión Global Precipitation Measurement (GPM) a la gestión integrada de los recursos hídricos en el Ecuador", y al Instituto Nacional de Meteorología e Hidrología (INAMHI).

\section{REFERENCIAS}

Alder, R., Bolvin, D., Huffman, G., Bolvin, D., Curtis, S., \& Nelkin, E. (2000). Tropical Rainfall Distributions Determinated Using TRMM combined with Other Satellite and Rain Gauge Information. Journal of Applied Meteorology, 39, 2007-2023.

Andrade, O. (2016). Evaluación de Imagenes satelitales de precipitación GPM (Global Precipitation Measurement) a escala sub-diaria para la provincia del Azuay. Cuenca: Tesis de Ingeniero Civil Universidad de Cuenca, 42.

Araghinejad, S. (2013). Data-Driven Modeling: Using MATLAB in Water Resources and Eviromental Engineering. In S. Ararghinejad, Data-Driven Modeling: Using MATLAB in Water Resources and Eviromental Engineering (Vol. 67, p. 290). Irán: Water Science and Technolgy library. 
Arteaga, M. O. (2017). Evaluación del modelo hidrológico hec-hms para la predicción hidrológica y de crecidas, en la cuenca baja del río Cañar. Quito: Tesis de Maestría en Recursos Hídricos Escuela Politécnica Nacional.

Ávila, Á. Justino, F., Wilson, A., Bromwich, D., \& Amorim, M. (2016) Recent precipitations trends, flash floods landslides in southern Brazil. Environmental Research Letters, 1-13. doi:10.1088/1748 9326/11/11/114029

Ayman, G., Nabil, A., \& Awdallah. (2013). A Novel Approach for the Join Use of Rainfall Monthly and Daily Gropund Station Data with TRMM Data to Generate IDF Estimates in a Poorly Gauged Arid Region. Open Journal of Modern Hydrology, 3, 1-7. doi:10.4236/ojmh.2013.31001

Azursa Véliz, F. A., Ramos Taipe, C., \& Lavado Casimiro, W. S. (2018, February 11). Evaluación de los productos Tropical Rainfall Measuring Mission (TRMM) y Global Precipitation Measurement (GPM) en el modelamiento hidrológicode la cuenca del río Huancané, Perú. Scientia Agropecuaria, 53-62. doi: 10.17268/sci.agropecu.2018.01.06

Ballari, D., Castro, E., \& Campozano, L. (2016). Validation of satellite precipitation (TRMM 3B43) in ecuadorian coastal plains, andean highlands and amazonian rainforest. XLI-B8, pp. 305-311. Prague, Czech Republic: Remote Sensing and Spatial Informations Sciences. Doi: 10.5194/isprs-archives-XLI-B8-305-2016

Bedient, P., Huber, W., \& Vieux, B. (2008). Hydrology and Floodplain Analysis. Houston, Texas: Prentice Hall.

Brunner, G. (2015). HEC-RAS River Analysis System Hydraulic. Davis, California: US Army Corps og Engineering.

Cabrera, E. (2016). Evaluación de imágenes satelitales de precipitaciónes GPM (Global Precipitation Measurement) a escala mensual para el Azuay. Cuenca: Tesis de Ingeniero Civil Universidad de Cuenca.

Castro, E. (2016). Evaluación de Imágenes Satelitales de Precipitaciónes(TRMM 3B43 mensual) en Región Costa, Sierra y Oriente del Ecuador. Cuenca: Tesis Ingeniero Civil, Universidad de Cuenca, 51.

CEPAL. (1984). Desastres Naturales 1982-1983 en Ecuador, Perú y Bolívia Quito: ONU, Comisión Económica para Latinoamérica.

Chinchilla, M., Mata, R., \& Alvarado, A. (2011). Andisoles, Inceptisoles Y Entisoles de la Subcuenca del Río Pirrís, Región de los Santos, Talamanca, Costa Rica. Agronomía Costarricense, 35 (1), 87-107.

Chow, V. T., Maidment, D., \& Mays, L. (1994). Hidrología Aplicada. Bogotá, Colombia: Editorial Nomos.

Córdova, E. (2017). Evaluación del prodcuto de precipitación diaria TMPA 3B42 V7 para su aplicabilidad en la modelización hidrológica en el Ecuador utilizando diferentes metodologías de corrección de datos. Quito, Ecuador: Tesis de Maestría en Recursos Hídricos, Escuela Politécnica Nacional.

Derin, Y., Anagnostou, E., Berne, A., Borga, M., Boudevillain, B., Buytaert, W., Yilmaz, K. (2016). Multiregional Satellite Precipitation Produtc Evaluation over Complex Terrain. Journal of Hydrometeorology, 17, 1817-1836. doi:10.1175/JHM-D-15-0197.1

Dinku, T., Hailemariam, K., Maidment, R., Tarnavsky, E., \& Connor, S. (2013). Combined use of satellite estimates and rain gauge observations to generate high-quality historical rainfall time series over Ethiopia. International Journal of Climatology, 16. doi: 10.1002/joc.3855

Duan, Z., Liu, J., Tou, Y., Chiogna, G., \& Disse, M. (2016). Evaluation of eight high spatial resolution gridded precipitation products in AdigeBasin(Italy) at multiple temporal andspacial scales. Science of the Total Environment, 573, 1536-1553. doi:10.1016/j.scitotenv.2016.08.213

Feldman, A. (2000). Hydrologic Modeling System Hec-HMs, Technical Reference Manual. U.S. Army Corps of Engineers, 145. Retrieved 2017 , from http://www.hec.usace.army.mil/publications/.

Feldman, A. (2000). Hydrologic Modeling System HEC-HMS: Technical Reference Manual. Washington: US Army Corps of Enginners.

Fleischbein, K., Wilcke, W., Goller, R., Boy, J., Valarezo, C., Zech, W., \& Knoblich, K. (2005). Rainfall interception in a lower montane forest in Ecuador: effects of canopy properties. Hydrological Processes, 19(7), 1355-1371. DOI:10.1002/hyp.5562.

Fleming, M., \& Doan, J. (2013). HEC-GeoHMS Geospatial Hydrologic Modeling Extensión: User's Manual. California: US Army Corps of Engineers.

Flemming, M., \& Brauer, T. (2016). Hydrologic Modelling System HEC_HMS Quick Start Guide. U.S. Army Corps of Engineers Institute of Water Resources Hydrologic Engineering Center (CEIWR-HEC), CPD-74D.

Guachamín, W., García, F., Arteaga, M., \& Cadena, J. (2015). Actualización estudio lluvias intensas. Determinación de Ecuaciones para el Cálculo de Intensidades Máximas de Precipitación. Actualización Estudio de Lluvias Intensas.
http://www.serviciometeorologico.gob.ec/Publicaciones/Hidrologia/EST UDIO_DE_INTENSIDADES_V_FINAL.pdf

Gupta, H., Sorooshian, S., \& Yapo, P. (1999). Status of automatic calibration for hydrological models: Comparison with multilevel expert calibration. 135-143.

Hingray, B., Picouet, C., \& Musy, A. (2015). Hydrology a Science for Engineers. France: Taylor \& Francis Group.

Horna, L. (2016). Evaluación Hidrometeorológica y de Sedimentos en la Cuenca del Río Coca en la Zona de Influenecia del Proyecto Coca Codo Sinclair. 125

Huffman, G. (2015, Noviembre 16). Algorithm Theoretical Basis Document (ATBD) Version 4.5, NASA Global Precipitation Measurement (GPM). Retrieved Septiembre 2017, from https://pmm.nasa.gov/sites/default/files/document_files/IMERG_ATBD _V4.5_0.pdf

Huffman, G. (2016, Octubre 25). The Transition in Multi-Satellite Products from TRMM to GPM (TMPA to IMERG). Retrieved from https://pmm.nasa.gov/sites/default/files/document_files/TMPA-toIMERG_transition_161025.pdf

Huffman, G., Adler, R., Bolvin, D., Gu, G., Nelkin, E., Bowman, K., Wolff, D. (2007). The TRMM Multisatellite Precipitation Analysis (TMPA): Quasi-Global, Multiyear Combined-Sensor Precipitation Estimates at Fine Scales. American Metorological Society, 8, 38-55. doi:10.1175/JHM560.1

INEC. (2015). Retrieved Julio 2016, from http://www.ecuadorencifras.gob.ec/category/cartografia-2/

Jha, A., \& Bloch, R. (2012). Vities and Flooding A Guide to Integrated Urban Flood Risk Management the 21st Century. Washington: The World Bank. Doi: 10.1596/978-0-8213-8866-2

Joo, J., Kjeldsen, T., Jun Kim, H., \& Lee, H. (2013). A comparision of two event-based flood models (ReFH-rainfal runoff model and HEC HMS) at two Korean catchments, Bukil and Jeungpyeong. KSCE Journal of Civil Engineering, 330-343. doi: 10.1007/s12205-013-0348-3

Li, M., \& Shao, Q. (2010). An improved statistical approach to merge satellite rainfall estimates and raingauge data. Journal of Hydrology (385), 51-64. doi:10.1016/j.jhydrol.2010.01.023

Liu, Z. (2015). Comparision of Integrated MUltisatellite Retrievals for GPM (IMERG) and TRMM Multisatellite Precipitation Analysis (TMPA) Monthly Precipitation Products:Initial Results. Jpurnal of Hydrometeorology, 17, 777-789. doi:10.1175/JHM-D-15-0068.1

MAE. (2012). Sistema de Clasificación de los Ecosistemas del Ecuador Continental. Quito: Subsecretaría de Patrimonio Natural.

Manz, B., Buytaert, W., \& Onof, C. (2014). Optimization of TRMM 2A25 Extreme Rainfall Rate and Probability. Geophysical Research Abstracts, 16,1

Manz, B., Buytaert, W., Zulkafli, Z., \& Lavado, W. (2016). High-resolution satellite-gauge merged precipitation climatologies of the Tropical Andes. Journal of Geophysical Research: Atmospheres, 121, 1-18. doi:10.1002/2015JD023788

Manz, B., Paez-Bimos, S., Horna, N., Buytaert, W., Ochoa, B., Lavado, W., \& Willems, B. (2017). Comparative Ground Validation of IMERG and TMPA at variable spatio-temporal scales in the Tropical Andes. Journal of Hydrometeorology, 18(9), 2469-2489. doi:10.1175/JHM-D-16-0277.1

Mena, S. (2010). Evolución dinámica de los escurrimientos en zonas de alta montaña: Caso del volcán Antisana. Quito: Titulo de Ingeniera Ambiental, Escuela Politécnica Nacional.

Méndez Rivas, R. A. (2016). Prdoductos de precipitación satelital de alta resolución espacial y temporal en zonas de topografía compleja. Tesis de Maestría, 1-98.

Menéndez, A., \& Re, M. (2009). Códigos Numéricos para Hidrología Urbana. Especialización en Hidrología Urbana (p. 134). Buenos Aires: Facultad de Ingeniería, UBA.

Moriasi, D., Arnold, J., Van Liew, M., Bingner, R., Harmel, R., \& Veith, T. (2007). Model evaluation guidelines for systematic quatification of accuracy in watershed simulation. Transactions of the ASABE, 50(3):885-900).

NASA. (2017). Precipitation Measurement Missions. Retrieved Octubre 01, 2017, from https://pmm.nasa.gov/TRMM/trmm-instruments

Nerini, D., Zulkafli, Z., Wang, L., Onof, C., Buytaert, W., Lavado, W., \& Guyot, J. (2015). A comparative analysis of TRMM-rain gauge data merging techniques at the daily time scale for distributed rainfall-runoff modelling applications. Journal of Hydrometeorology, 1-42. doi: 10.1175/JHM-D-14-0197.1

Nikolopoulos, E., Anagnostou, E., \& Borga, M. (2013). Using HighResolution Satellite Rainfall Products to Simulate a Major Flash Flood 
Event in Northern Italy. Journal of Hydrometeorology, 14, 171-185 doi:10.1175/JHM-D-12-09.1

NRCS. (1986). Urban Hydrology for Small Watersheds TR-55. Washington DC: The U.S Department of Agriculture (USDA).

Nuñez, J. (1983). Fundamentos de Edafología. (2nd Edi., 13, 37, 41 pp.) San José, Costa Rica: Editorial EUNED.

OMM. (2006). Aspectos Ambientales de la Gestión Integrada de Crecidas. Ginebra, Suiza: APFM.

OMM. (2009). Aspectos sociales y participación de los interesados en la gestión integrada de crecidas. Ginebra: OMM.

OMM. (2011). Guía de prácticas hidrológicas, Gestión de Recursos hídricos y aplicación de prácticas hidrológicas. Organización Meteorológica Mundial (168).

Páez Bimos, S., \& Llerena, S. (2014). Sistema de alerta temprana hidrometeorológico para inundaciones por crecidas de ríos cuenca del río Cañar (SAT Cañar), Manual de operación. Quito: INAMHI.

Pérez, K. (2013). Modelación hidrodinámica para la implementacion del Sistema de Alerta Temprana en la cuenca del río Cañar. Quito: INAMHI.

Pérez, K., Ordoñez, F., Hinojosa, D., \& Guachamín, W. (2013). Definición de umbrales y presentación de resultados para la implementación del sitema de alerta temprana en la cuenca del río Cañar. Quito: INAMHI.

Prakash, S., Mitra, A., Pai, D., \& AghaKouchak, A. (2016). From TRMM to GPM:How well can heavy rainfallbe detected from space? Advances in Water Resources, 88, 1-7. doi:http://dx.doi.org/10.1016/j.advwatres.2015.11.008

Razi, M., Ariffin, J., Tahir, W., \& Arish, N. (2010). Flood estimation studies using hydrologic modeling system (HEC-HMS) for Johor River, Malaysia. Journal of Applied Sciences, 930-939. doi:10.3923/jas.2010.930.939

Satgé, F., Bonnet, M., Gosset, M., Molina, J., Yuque, W., Pillco, R., Garnier, J. (2015). Assessment of satellite rainfall products over teh Andean plateau. Atmospheric 1-14. doi:10.1016/j.atmosres.2015.07.012

Sedano, K., Ávila, Á., \& Carvajal, Y. (2013). ANÁLISIS DE ASPECTOS QUE INCREMENTAN EL RIESGO DE INUNDACIONES EN COLOMBIA. Luna Azul, 37(ISSN 1909-2474), 219-238.

Shahid, M., Boccardo, P., Usman, M., Albanese, A., \& Qamar, M. (2016). Prdicting Peak Flows in Real Time through Event Based Hydrologic Modeling for a Trans-Boundary. Water Resour Manage, 793-810.

Sharifi, E., Steinacker, R., \& Saghafian, B. (2016). Assessment of GPMIMERG and Other Precipitation Products against Gauge Data under Different Topographic and Climatic Conditions in Iran. Remote Sens, 135, 1-24. doi:10.3390/rs8020135

Shen, Y., Xiong, A., Wang, Y., \& Xie, P. (2010). Perfomance of hinghresolution satellite precipitation products over China. Journal of Geophysical Research, 115, 1-17. doi:10.1029/2009JD012097

Tan, J., Petersen, W., \& Tokay, A. (2016). A Novel Approach to Identify Sources of Errors in IMERG to GPM Ground Validation. Journal of Hydrometeorology, 1-44. doi:10.1175/JHM-D-16-0079.1

Tang, G., Ma, Y., Long, D., Zhong, L., \& Hong, Y. (2016). Evaluation of GPM Day-1 IMERG and TMPA version-7 legacy products over Maimland China at multiple spatiotemporal scales. Journal of Hydrology, 533, 152-167. doi:10.1016/j.jhydrol.2015.12.008

Tang, G., Zeng, Z., Long, D., \& Guo, X. (2016). Statistical and Hydrological Comparisons between TRMM and GPM level-3 Products over a Midlatitude Basin: Is Day-1 IMERG a Good Successorfor TMPA 3B42V7? Journal of Hydrometeorology, 17, 121-137. doi:10.1175/JHMD-15-0059.1

Thompson, L., \& Troeh. (2002). Los Suelos y su Fertilidad. Sevilla, España: Editorial REVERTÉ.

USDA. (1999). Soil Taxonomy. Washington, DC: U.S. Government Printing Office.

Viessman, W., \& Lewis, G. (2003). Introduction to Hydrology. (5ta Edi. 612 p.) Baltimore, Maryland: Pearson Education Inc.

Ward, E., Buytaert, W., Peaver, L., \& Wheater, H. (2011). Evaluation of precipitation products over complex mountainous terrain: A water resources perspective. Advaces in Water Resources, 1222-1231. doi:10.1016/j.advwatres.2011.05.007

Xuefeng, C., \& Steinman, A. (2009). Event and Continuous Hydrologic Modeling with HEC-HMS. Journal of Irrigation and Drenage Engineering, 135 (1) 119-124.

Yang, Y., Du, J., Cheng, L., \& Xu, W. (2017). Applicability of TRMM satellite precipitation in driving hydrological model for Identifying flood events: a case study in the Xiangjiang River Basin, China. Nat Hazards, 1489-1505. doi: 10.1007/s11069-017-2836-0
Zubieta, R., Getirana, A., Espinoza, J., Lacado Casimiro, W., \& Aragon, L. (2017). Hydrological modeling of the Peruvian-Ecuadorian Amazon Basin using GPM-IMERG satellite-based precipitation dataset. Hydrology and Earth System Sciences, 2543-3555. doi:10.5194/hess-213543-2017

Zulkafli, Z., Buytaert, W., Onof, C., Manz, B., Tarnavsky, E., Lavado, W., \& Guyot, J. (2014). A Comparative Performace Analysis of TRMM 3B42 (TMPA) Versions 6 and 7 for Hydrological Aplications over AndeanAmazon River Basins. Journal of Hydrometeorology, 15, 581-592. doi:10.1175/JHM-D-13-094.1

\section{BIOGRAFÍAS}

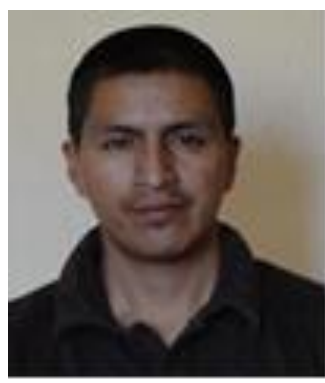

\begin{abstract}
Wilmer Guachamín Acero. Ingeniero Agrónomo en la Universidad Central del Ecuador, Magister en Ambiental de la Escuela Politécnica Nacional, Quito-Ecuador. En la actualidad se desempeña en Estudios, Investigación y Desarrollo Hidrometeorológico en el Instituto Nacional de Meteorología e Hidrología (INAMHI).
\end{abstract}

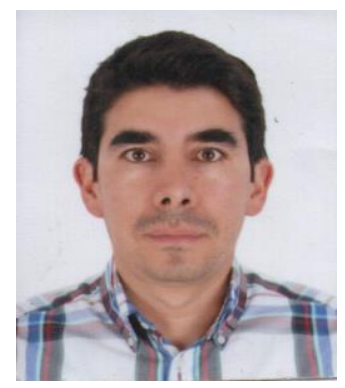

Sebastián Páez-Bimos. Ingeniero Civil graduado en la Pontificia Universidad Católica del Ecuador. Master of Science en Water Resources Engineering and Management de la Universitaet Stuttgart - Alemania. Profesor titular de la Facultad de Ingeniería Civil y Ambiental de la Escuela Politécnica Nacional. Sus intereses de investigación se enfocan en el área de los recursos hídricos, hidrometeorología y modelación hidrológica. Actualmente es doctorante en ciencias de la Université Catholique de Louvain - Bélgica donde estudia el comportamiento de los flujos de agua y solutos en el páramo andino.

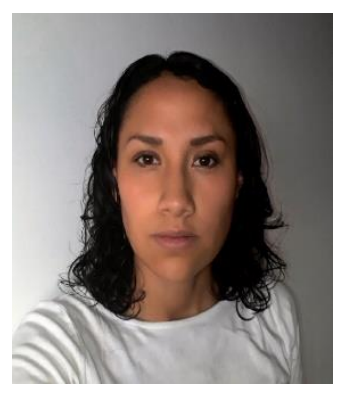

Luisa Natalia Horna Kuonqui. Ingeniera Civil mención Hidráulica graduada en la Escuela Politécnica Nacional. Su tesis la desarrolló en el marco de un proyecto de investigación en la cuenca del río Coca. Ha colaborado en la evaluación de productos de precipitación satelital en varias zonas de estudio. Actualmente trabaja en la Dirección de Estudios, Investigación y Desarrollo Hidrometeorológico del INAMHI. 